This is the final peer-reviewed accepted manuscript of:

Negroni, G., \& Bagnoli, L. (2017). On the coevolution of social norms in primitive societies. Journal of Economic Interaction and Coordination, 12(3), 635-667.

The final published version is available online at:

https://doi.org/10.1007/s11403-016-0180-1

Rights / License:

The terms and conditions for the reuse of this version of the manuscript are specified in the publishing policy. For all terms of use and more information see the publisher's website. 
Noname manuscript No.

(will be inserted by the editor)

\title{
On the coevolution of social norms in primitive societies
}

\author{
Giorgio Negroni · Lidia Bagnoli
}

Received: date / Accepted: date

\begin{abstract}
We study the evolutionary origin of a social norm of the kind "cooperate frequently and share fully" observed in modern hunter-gatherers. In order to do this, a two-stage game in which a pie has first to be produced and then divided is proposed. We assume that the bargaining rule is sensitive to investment behavior and to the degree of property rights protection. We show that, when a unique stochastically stable outcome exists, a norm of investment and a norm of division coevolve supporting the efficient investment profile and the egalitarian distribution of the surplus, respectively. The conditions needed for norms to coevolve depend on whether property rights over the fruits of one's own independent investment are secured or not.
\end{abstract}

Keywords Evolution - Social norms · Stochastically stable equilibrium • Modern hunter-gatherer societies

JEL classification: C78, D83, Z13

G. Negroni

Department of Economics, University of Bologna, Strada Maggiore 45, 40125 Bologna, Italy Tel.: +39-543-374110, Fax: +39-0543-374119

E-mail: giorgio.negroni@unibo.it

L. Bagnoli

Ministry of Economy and Finance, via Solferino 11, 47121 Forlì, Italy 


\section{Introduction}

We know from the anthropological literature that in modern hunter-gatherers food acquisition and food distribution are regulated by social norms (Boehm, 2012). A neat example can be found among the Ache of Paraguay who follow a rule of thumb for hunted resources of the kind "cooperate frequently and share fully" (Hill, 2002). Social norms of this kind can also be found among the whale hunters in Lamalera (Alvard and Nolin, 2002), the Mbuti pygmies (Ichikawa, 1983) the Hazda, the !Kung (Woodburn, 1982), among others.

Despite the empirical evidence on the widespread use of social norms in primitive societies, our knowledge of their origin is still limited; according to Kaplan and Gurven (2005), in fact, "we know virtually nothing about how standards for appropriate behavior emerge and change in small-scale societies without official means of enforcement" (p. 96).

Binmore (2005) put forward the idea that standards of behavior evolved to solve the equilibrium selection problem embedded in the repeated game of life played by our ancestors. Anthropologists suggest that the relevant game of life was large-game hunting (Boehm, 2004, 2012). The idea is that a dietary shift towards high quality food packages started after the discovery of largegame hunting. However, these food packages were difficult and dangerous to acquire by a solitary hunter. Acknowledgement of this fact probably increased the benefit of collective action and facilitated sharing (Kaplan and Gurven, 2005).

The present paper is an attempt to study the evolutionary origin of a social norm of the kind "cooperate frequently and share fully". Recent papers by Troger (2002), Ellingsen and Robles (2002) and Dawid and MacLeod (2001) provide a useful framework of analysis. They are concerned with a two riskneutral players, two-stage game in which a pie has first to be produced (stage one) and then divided (stage two). ${ }^{1}$ In particular, while Troger (2002) and Ellingsen and Robles (2002) showed that evolution supports the efficient equilibrium when the pie is determined by the decision of one agent only, Dawid and MacLeod (2001) proved instead that this efficiency result may not hold when the pie depends on the decisions of both agents. ${ }^{2}$

This literature, however, is not totally adequate to explain the evolutionary origin of social norms observed in primitive societies. On one side, in fact,

\footnotetext{
1 This literature is concerned with evolutionary dynamics in scenarios characterized by investment specificity. We say that investment is completely relation-specific when it is only valuable to a particular trading partner. By making investor vulnerable to ex-post exploitation, investment specificity may thus give rise to the so-called hold-up problem. Relation-specific investment seems a minor problem for nomadic hunter-gatherers since, for their subsistence, they are not dependent on specific other agents, nor on specific assets and resources; see Woodburn (1982).

2 Other important contributions include Young (1993) and Hawkes (1992). The former explains the evolution of a division norm when the pie is exogenously given; the latter explains the evolution of a norm regulating agents' cooperation in producing a pie, when the division rule is exogenously given.
} 
Troger (2002) and Ellingsen and Robles (2002) do not give strong support to the egalitarian surplus division ${ }^{3}$, which is instead a distinguishing feature of hunter-gatherers; moreover, their assumption that the pie only depends on the decision of one agent seems quite restrictive. On the other hand, although Dawid and MacLeod (2001) made an important step by removing this limiting assumption ${ }^{4}$, their way of shaping the distribution stage is not totally convincing. Sure enough, when we allow both agents to strategically interact in producing a pie, we have to specify which division protocol is adequate at each possible division node. Dawid and MacLeod (2001) assume that a Nash Demand Game (NDG) occurs after an asymmetric investment profile while equal split occurs after a symmetric investment profile. They defend the equal division assumption by appealing to Young (1993) who proved this result for an exogenously given pie. However, since in Dawid and MacLeod (2001) the pie to be divided is not exogenously given, the equal split assumption seems not adequate because it sweeps off the main issue.

In this paper we consider a two-stage game in which the pie depends on the decisions of two risk neutral agents, as in Dawid and MacLeod (2001). However, we depart from their framework in two respects.

Firstly, we posit that in each period our game is played by two agents belonging to two different populations, $A$ and $B$, of equal size. This allows us to apply the evolutionary framework for extensive games put forward by Noldeke and Samuelson (1993). ${ }^{5}$ The assumption of two populations can be easily justified in our model; it corresponds, for instance, to a situation in which each member of the community is appointed with a portion of the communal land for his activity.

Secondly, we posit that the bargaining game played in the second stage is sensitive to the investment profile observed and to the degree of property rights protection. We assume that when both agents invest in producing a pie, they are involved in a NDG; when instead nobody invests there is no bargaining since there is no surplus. What becomes critical is the bargaining occurring when an asymmetric investment profile is observed, that is when only one invests in producing a pie. Here two different scenarios are considered, depending on whether property rights over the fruits of one's own independent investment are secured or not.

\footnotetext{
3 More precisely, they show that the evolved division norm virtually assignes the entire surplus to the unique investing agent, provided that a fine grid of investment choices is allowed.

4 Dawid and MacLeod (2008) is a further extension in which the outcome of the investment decision is stochastic.

5 This is not the evolutionary framework considered by Dawid and MacLeod (2001) who are basically concerned with an adaptation of Young (1993a) to an extensive form game. Athough this extension is not problematic with one-sided investment (as in Troger 2002), it is a bit tricky with two-sided investment since it can imply that some agents continue to believe that all the opponents make the same investment (i.e. all choose high or low investments) even when some bargaining outcomes (which in their model can only happen when high-low matches occur) are observed.
} 
When property rights are secured, we explore the case in which the bargaining stage is described by a Dictator Game (DG) in which the unique investing agent has full control of the surplus division; in this case if a sharing occurs it only depends on the free will of the investing agent.

When instead property rights are not secured, we explore the case in which the bargaining stage is described by an Ultimatum Game (UG) in which the unique investing agent proposes a division which is realized only if accepted by the opponent. Here we have in mind a situation in which, since the fruits of one's own independent investment are considered communal property, people feel entitled to a fair share of it; as a consequence, not only the unique hunting agent cannot refuse to share with the not participant one, but he must also share fairly, according to society's rule. The UG captures the fact that any violation of this standard may result into overt conflict and consequent loss of the carcass. ${ }^{6}$

We are thus concerned with two different two-stage games, that we label $\Gamma_{D G}$ and $\Gamma_{U G}$, which only differ in the bargaining rule following an asymmetric investment profile. We claim that a standard of behavior of the kind "cooperate frequently and share fully" in use among modern hunter-gatherers probably evolved from structures of interactions somehow amenable to one of these two games. Of course, other alternatives could be considered and we leave to further research to investigate the robustness of our results.

Evidence from anthropology suggests that both situations (i.e. private and communal property over the fruits of one's own independent production) are indeed plausible. For instance, Hill (2002) reports that among the Ache only the individual who has participated in attempting to acquire resources is entitled to bargaining over a share of those resources, thus making sharing contingent upon contribution; in this case, those who do not contribute are not entitled to a share. However, Boehm (2004) says that individuals living in small nomadic bands often do not have the right to treat the carcasses they have killed as private property; rather, in these cases, "the entire band is taking over what otherwise would be private property of one or more successful hunters, and is treating it as group-wide communal property because everyone, including those not active in procuring the carcass, will insist on sharing in the commodity" (p. 118). Analogously, Woodburn (1982) reports that among

\footnotetext{
6 In a preliminary study we considered the case in which a NDG, rather then an UG, occurs after an asymmetric investment profile; however we were not able to derive the stochastically stable equilibrium due to the occurrence of several not singleton absorbing sets with complex structure. Ellingsen and Robles (2002) also considered the case in which, in stage two, the distribution of the surplus is determined by an ultimatum game where the player who makes the proposal is the trading partner, i.e. the agent not responsible for the investment decision. They have shown that in this case the stochastic stability has little cutting power because many outcomes are stochastically stable. Our game $\Gamma_{U G}$ mainly differs from Ellingsen and Robles (2002) in two respects. First, the player who makes the proposal is the player who decided to invest in the first stage. Second, since the pie depends on the decisions of two agents, both can be in a position to affect the distribution of the surplus generated by the other. Our result for $\Gamma_{U G}$ says that, under the appropriate conditions, a unique stochastically stable outcome exists.
} 
the !Kung and the Hazda, sharing is not contingent upon contribution since "some men who are regular recipients never themselves contribute" and argues that this distribution must be considered as "socially imposed" on the solitary hunter, as a form of modern taxation. ${ }^{7}$ While the situation described by Hill (2002) seems compatible with $\Gamma_{D G}$, the evidence reported by Boehm (2004) and Woodburn (1982) seems compatible with $\Gamma_{U G}$.

Both games have a multiplicity of equilibria. In order to identify the evolved social norms we use the concept of stochastic stability. We claim that a social norm has evolved when the stochastically stable set only supports an homogeneous behavior for at least one population. Our main result states that when a social norm evolves then not only we observe an homogeneous behavior along the whole path of play but the prescribed actions are uniform across populations. We can thus claim that, in both games, norms coevolve: the investment norm supports full cooperation in the production stage (meaning that both agents choose to invest in producing a pie) and the bargaining norm entails an egalitarian division of the realized surplus. Our main result says that a social norm of the kind "cooperate frequently and share fully" evolves in both games.

The two games differ for the in demand conditions for norms to coevolve. In particular, we always observe a coevolution of norms in $\Gamma_{D G}$; the intuition behind this result is that a single investor will appropriate the entire small pie, so that the best the other can hope for is zero (or the least feasible division). In $\Gamma_{U G}$ instead the coevolution of social norms only occurs when investments are complements and the cost of investment is not too high. The intuition now is that equal sharing evolves when there are large surplus gains ${ }^{8}$; in this case, in fact, the gross surplus obtained when both agents invest is so large that dividing it equally yields to each agent more than the maximum payoff one can hope to get when she is the only investing agent. However, when these conditions fail a great deal of outcomes are stochastically stable so that neither an investment norm nor a bargaining norm evolve. ${ }^{9}$

Our main results are derived under the natural assumption that full cooperation in the production stage is efficient; this means that the net surplus observed when both agents choose to invest is greater than the net surplus

\footnotetext{
7 Woodburn (1982) suggests that among the Hazda and the !Kung this "right to a share" by those not investing is responsible for the overwhelming difficulties encountered by those who make an effort to live by agriculture.

8 The connection between complementarity and equal sharing is also suggested, albeit implicitly, by Boehm (2012). He maintains that team hunting is an energetically demanding occupation which works best if all the members of the hunting team are adequately nourished, a condition allowed by equal sharing.

9 It is worth observing that, although the formal conditions for a stochastically stable outcome to exist in $\Gamma_{U G}$ coincide with those required by Dawid and MacLeod (2001), the basic models and the evolutionary dynamics are different. In particular, while in $\Gamma_{U G}$ these conditions support the coevolution of a norm of cooperation and a norm of division, they only uphold a norm of investment in Dawid and MacLeod (2001).
} 
when only one invests. Recently Avard (2004) have suggested the hypothesis that standards of cooperation and fair division are more likely to be observable in societies in which "the payoffs to cooperation are high". In order to verify Alvard's hypothesis, in the last Section we very briefly extend our analysis to the case in which full cooperation in the production stage is not necessarily efficient and we show that our results do not change.

The basic model is presented in Section 2. Section 3 describes the evolutionary dynamics and gives some preliminary results. The main results are provided in Section 4, and further insights are discussed in Section 5.

\section{The model}

Two risk neutral players $(A$ and $B)$ are engaged in a two-stage game. In stage one both have to simultaneously decide whether to invest (action $H$ ) or not (action $L$ ) in producing a pie. The investment cost $(c)$ is identical for both agents. When both choose $H$ the gross surplus is $V_{H}$; when only one chooses $H$ the gross surplus is $V_{M}$; when both choose $L$ there is no surplus. Obviously, $V_{H}>V_{M}>0$. At the end of stage one the produced surplus is observed and each player can correctly estimate her opponent's choice. In stage two they bargain over the available surplus. If both invest, they are engaged in a Nash Demand Game (NDG). If only one invests, the paper explores two possibilities: either an Ultimatum Game (UG) or a Dictator Game (DG).

We denote by $\Gamma_{U G}\left(\right.$ resp. $\left.\Gamma_{D G}\right)$ the whole extensive game in which a NDG occurs when both players invest and a UG (resp. DG) occurs when only one invests. In NDG players $A$ and $B$ simultaneously make demands $y$ and $x$, respectively. If these demands are compatible, each receives what she claimed; otherwise they receive nothing. Since agents are risk neutral, the payoffs are $\pi_{A}=y-c$ and $\pi_{B}=x-c$ if $y+x \leq V_{H} ; \pi_{A}=-c$ and $\pi_{B}=-c$ if $y+x>V_{H}$. In UG the player who invests makes a proposal which the opponent can either accept or reject. In DG the division continues to be advanced by the player who invests; however her opponent now has no choice but to accept. Let $D\left(V_{j}\right)=\left\{\delta, 2 \delta, \ldots, V_{j}-\delta\right\}, j \in\{H, M\}$ denote the set of feasible claims.

Throughout the paper we make the following assumption:

Assumption 1 (a) $\frac{V_{H}}{2}$ and $c$ are divisible by $\delta$ and $c>\delta$;

(b) the efficient net surplus arises when both players choose $H$, i.e.

$$
c<\min \left(\frac{V_{H}}{2} ; V_{H}-V_{M}\right) ;
$$

(c) the maximum payoff attainable by playing $H$ when the opponent chooses $L$ is not negative, i.e.

$$
c \leq V_{M}-\delta
$$


It is worth noting that, under Assumption 1, both $\Gamma_{U G}$ and $\Gamma_{D G}$ admit a subgame perfect equilibrium which supports investment profile $H H$. Nevertheless, both games admit a great deal of subgame perfect equilibria some of which are inefficient.

\section{Evolutionary dynamics}

In this Section we embed our model into the evolutionary framework put forward by Noldeke and Samuelson (1993). To this end we postulate a finite population of size $N$ for each player, $A$ and $B$. In each period, every possible match between agents occurs meaning that each agent belonging to population $A$ interacts with each agent of population $B$, one at a time. An agent is described by a characteristic which consists of a detailed plan of action and a set of beliefs about the opponent's behavior. ${ }^{10}$

Let $\theta$ be a state; this denotes a profile of characteristics of the overall population. $z(\theta)$ denotes the probability distribution across the terminal nodes generated by $\theta$; lastly, $\Theta$ is the finite set of possible states.

At the end of every period each agent with probability $\lambda$ observes $z(\theta)$ and her characteristic may change. In particular the received information allows agents to correctly update their beliefs on opponent's choices at the observed information sets. Given their new beliefs, they also update their action profile by choosing a best reply ${ }^{11}$ at each information set. With probability $1-\lambda$ the single agent does not observe $z(\theta)$ and her characteristic does not change. This learning mechanism engenders an (unperturbed) Markov process $(\Theta, P)$ where $P$ is the transition matrix on $\Theta$.

By $\Omega$ we denote a generic limit set ${ }^{12}$ of the process; this is a minimal subset of states such that, when the process enters, it does not exit. By $\rho(\Omega)$ we denote the set of observable terminal nodes under $\Omega$. Lastly by $\Sigma$ we denote the union of the limit sets of the process.

Besides being updated, agents' beliefs and actions can also change by mutation. In every period, each agent has a probability $\epsilon$ of mutating. Mutations are independently distributed across agents. When mutating, agent changes her characteristic according to a probability distribution assigning positive probability on each possible characteristic.

\footnotetext{
10 In $\Gamma_{U G}$, a plan of action for player $A$ must specify: (i) the type of investment; (ii) the demand when both players choose $H$ (the action at $H H$ ); (iii) the demand when $A$ chooses $H$ and $B$ chooses $L$ (the action at $H L$ ); (iv) whether to accept or reject any demands made by $B$, when in the first stage $B$ chooses $H$ and $A$ chooses $L$. The same applies for player $B$. In $\Gamma_{D G}$, a plan of action for player $A$ must specify: (i) the type of investment; (ii) the demand when both players choose $H$ (the action at $H H$ ); (iii) the division of the surplus when $A$ chooses $H$ and $B$ chooses $L$ (the action at $H L$ ). Analogously for player $B$.

11 However, if the learning agent has already played a best reply her action does not change. Moreover, when the best reply contains more than one action, one of these can be randomly chosen according to a distribution with full support.

12 A set $\Omega \subseteq \Theta$ is called a limit set of the process $(\Theta, P)$ if: (a) $\forall \theta \in \Omega$, $\operatorname{Prob}\left\{\theta_{t+1} \in \Omega \mid \theta_{t}=\theta\right\}=1$; (b) $\forall\left(\theta, \theta^{\prime}\right) \in \Omega^{2}, \exists s>0$ s.t. $\operatorname{Prob}\left\{\theta_{t+s}=\theta^{\prime} \mid \theta_{t}=\theta\right\}>0$.
} 
A particular type of mutation occurs when the mutants change belief and/or action at some unreached information set under the current state $\theta$. In this case we assert that the state drifts.

Mutations generate a new (perturbed) Markov process $(\Theta, P(\epsilon))$, which is ergodic. It is well known that, for any fixed $\epsilon>0$, the perturbed process has a unique invariant distribution $\mu_{\epsilon}$. Let $\mu_{*}=\lim _{\epsilon \rightarrow 0} \mu_{\epsilon}$ denote the limit distribution. A state $\theta$ is stochastically stable if $\mu_{*}(\theta)>0$. We denote the set of stochastically stable states by $\Sigma_{S}$; this is the set of states which has a positive probability in limit distribution. Noldeke and Samuelson (1993) proved that the stochastically stable set is contained in the union of the limit sets of the unperturbed process. In order to detect the stochastically stable set we first have to characterize the limit sets of our model; this is the aim of the following two Propositions.

Proposition 1 In $\Gamma_{U G}$ all the limit sets have one of the following structures: (a) they contain one state only, and this is a self-confirming equilibrium of the game; (b) they contain more than one state and all investment profiles are observed. Moreover, only one outcome is realized for each investment profile in which the claims exhaust the surplus.

Proof. See the Appendix

Proposition 2 In $\Gamma_{D G}$ all the limit sets contain one state only, and this is a self-confirming equilibrium. Moreover, at least one agent chooses to invest at every equilibrium.

Proof. See the Appendix

From now on when we speak of equilibrium we refer to self-confirming equilibrium..$^{13}$

Propositions 1 and 2 state that the considered evolutionary dynamic gives rise to a large multiplicity of limit sets. However, this dynamic admits limit sets in which both investment and bargaining behavior is uniform in each population. It is thus likely that homogeneous behavior in one or both populations could be molded by evolution. When this happens, we say that a norm has evolved. Accordingly, an investment norm has evolved if all agents belonging to the same population make the same investment and the investment behavior is correctly anticipated. Analogously, a bargaining norm has evolved if a pair of demands $(y, x)$ exists at some reached information set which exhausts the gross surplus and the bargaining behavior is correctly anticipated. When the set of stochastically stable states contains only equilibria supporting the same outcome we speak of a stochastically stable outcome rather than a stochastically stable set.

\footnotetext{
13 According to Noldeke and Samuelson (1993) a state is a self-confirming equilibrium if each agent's strategy is a best response to that agent's conjecture and if each agent's conjecture about opponent's strategies matches the opponent's choices at information sets that are reached in the play of some matches.
} 
Despite the fact that Propositions 1 and 2 do not help to pin down which behavior is more likely to become the conventional one, in the next Section we shall show that the stochastically stable set can support only one outcome in both games. Our remarkable result stems from direct application of Noldeke and Samuelson (1993) and Ellison (2000). In particular, Proposition 1 of Noldeke and Samuelson (1993) states that if $\Sigma_{S}$ is a strict subset of $\Sigma$ (i.e. $\left.\Sigma_{S} \subset \Sigma\right)$ then a $\Omega \notin \Sigma_{S}$ can not be reached from $\Sigma_{S}$ by a sequence of singlemutation transitions. Hence, our first task (Lemma 1 and 2 below) is to detect the smallest $\widehat{\Sigma} \subset \Sigma$ such that a sequence of a single-mutation transitions is enough to escape from any $\Omega \in \Sigma \backslash \widehat{\Sigma}$ and reach $\widehat{\Sigma}$. In this case when $\Sigma_{S} \subset \Sigma$ we know that $\Sigma_{S} \subset \widehat{\Sigma}$.

The next Lemma states our first preliminary result, which holds true for both the extensive games considered.

Lemma 1 Consider a limit set $\Omega$ such that $\rho(\Omega)$ is not a singleton. An equilibrium supporting one outcome only can be reached from $\Omega$ by a sequence of single-mutation transitions.

Proof. See the Appendix.

Lemma 1 enables us to limit our attention to the equilibria supporting one outcome only. According to the investment profile observed, we can partition this set of equilibria into four subsets. We denote these subsets respectively as $\Sigma_{H}, \Sigma_{L}, \Sigma_{H L}$ and $\Sigma_{L H}$. Of course, $\Sigma_{H}$ includes all the equilibria supporting the outcome $\left\{H H, V_{H}-x_{H H}, x_{H H}\right\}$ where $x_{H H} \in D_{\delta}\left(V_{H}\right)$. The same applies for the other subsets. The following Lemma 2 highlights that the process can move from any equilibrium belonging to the set $\left(\Sigma_{L} \cup \Sigma_{H L} \cup \Sigma_{L H}\right)$ to a new equilibrium $\theta \in \Sigma_{H}$ through a sequence of single-mutation transitions.

Lemma 2 Consider an equilibrium $\theta$; then:

(a) if $\theta \in \Sigma_{L}$ an equilibrium $\theta^{\prime} \in \Sigma_{H}$ can be reached from $\theta$ by a sequence of single-mutation transitions provided that $c+\delta<x_{H H}<V_{H}-c-\delta$;

(b) if $\theta \in \Sigma_{H L}$ (resp. $\Sigma_{L H}$ ) an equilibrium $\theta^{\prime} \in \Sigma_{H}$ can be reached from $\theta$ by a sequence of of single-mutation transitions provided that $c+\delta<x_{H H}<$ $V_{H}-c-\delta$.

Proof. See the Appendix.

It is worth stressing that since subset $\Sigma_{L}$ is empty in $\Gamma_{D G}$, then only point (b) of the previous Lemma is relevant to this game.

Lastly Lemma 3 below asserts that in $\Gamma_{U G}$ a sequence of single-mutation transitions is enough to shift the process from any equilibrium $\theta \in \Sigma_{L}$ to a new equilibrium $\theta^{\prime}$ in which only one agent has invested.

Lemma 3 Consider $\Gamma_{U G}$ and an equilibrium $\theta \in \Sigma_{L}$; an equilibrium $\theta^{\prime} \in$ $\Sigma_{L H} \cup \Sigma_{H L}$ can be reached from $\theta$ by a sequence of single-mutation transitions provided that at $\theta^{\prime}$ the agent who has chosen $H$ is better off; 
Proof. See the Appendix.

All the results so far obtained only require that $c<\min \left(V_{M}, \frac{V_{H}}{2}\right)$. From Lemma 1 we know that, when a limit set underpins a multiplicity of outcomes, then we can reach an equilibrium sustaining only one outcome by a sequence of single mutations. Lemma 2 tells us that if the single equilibrium outcome does not support the efficient investment profile, then the process can reach a single equilibrium belonging to $\Sigma_{H}$ by a sequence of single mutations. Hence, when $\Sigma_{S} \subset \Sigma$, both these Lemma suggest we focus on subset $\Sigma_{H}$.

\section{Main results}

Given the results so far disclosed, in this Section we limit our concern to the set of equilibria $\Sigma_{H}$ in order to explore, for both games, the occurrence of some norms. First and foremost we provide the characterization of the stochastically stable set for $\Gamma_{U G}$; we then briefly consider $\Gamma_{D G}$. To simplify our analysis we introduce the following mild technical assumption, which holds for both games.

Assumption 2 The population is sufficiently large, i.e. $\frac{V_{H}}{N}<\delta$.

Consider $\Gamma_{U G}$ and let $x_{B}^{U}$ (resp. $V_{H}-x_{A}^{U}$ ) be the share going to player $B$ (resp. $A$ ) such that she receives a payoff equal to $V_{M}-\delta$ when both agents have invested:

$$
\begin{aligned}
& x_{B}^{U}=V_{M}-\delta+c \\
& x_{A}^{U}=V_{H}+\delta-c-V_{M} .
\end{aligned}
$$

Since $c$ is divisible by $\delta$ and $c>\delta$, then $x_{B}^{U}, x_{A}^{U} \in D_{\delta}\left(V_{H}\right)$. Let ${ }^{14}$

$$
\begin{aligned}
& \widehat{x}_{A}^{U}=\max \left\{x \in D_{\delta}\left(V_{H}\right) \mid\left(V_{H}-x\right) \frac{N-1}{N}-c \geq V_{M}-\delta\right\} \\
& \widehat{x}_{B}^{U}=\min \left\{x \in D_{\delta}\left(V_{H}\right) \mid x \frac{N-1}{N}-c \geq V_{M}-\delta\right\} .
\end{aligned}
$$

Under assumption 2 it follows that $\widehat{x}_{A}^{U}=x_{A}^{U}-\delta$ and $\widehat{x}_{B}^{U}=x_{B}^{U}+\delta$. Therefore $\widehat{x}_{B}^{U} \leq \widehat{x}_{A}^{U}$ if

$$
V_{M} \leq \frac{V_{H}}{2}-c
$$

When this condition holds, then we can define the following set:

$$
\Sigma_{I H}^{U}=\left\{\theta \in \Sigma_{H} \mid x \in\left[\widehat{x}_{B}^{U}, \widehat{x}_{A}^{U}\right]\right\} .
$$

It is worth noticing that $V_{H}-\widehat{x}_{A}^{U}=\widehat{x}_{B}^{U}$, meaning that in $\Sigma_{I H}^{U}$ the lowest share of surplus is the same for both agents. Few computations show that $\frac{V_{H}}{2} \in \Sigma_{I H}^{U}$.

By definition when $\theta \in \Sigma_{I H}^{U}$ each agent receives an equilibrium payoff not smaller than the maximum payoff attainable when she deviates by playing $L$.

\footnotetext{
$14 \widehat{x}_{A}^{U}$ is the largest demand agent $B$ can make at $H H$ such that $A$ does not have any incentive to change action by playing $L$ when she knows that: (i) $N-1$ agents $B$ play $H$ and claim $\widehat{x}_{A}^{U}$; (ii) one agent $B$ makes a larger demand. Analogously for $\widehat{x}_{B}^{U}$.
} 
Any equilibrium in $\Sigma_{I H}^{U}$ thus dominates all the equilibria supporting other investment profiles. Hence even if at an equilibrium $\theta \in \Sigma_{I H}^{U}$ the belief on the outcome in high-low matches drifts, allowing some agents to expect to get almost the whole surplus if they do not invest, this drift does not push the process away from the basin of attraction of $\theta$.

We can always partition the set $\Sigma_{H}$ into $\Sigma_{I H}^{U}$ and $\Sigma_{C H}^{U}=\Sigma_{H} \backslash \Sigma_{I H}^{U}$ where the latter denotes the set of equilibria in which both agents choose to invest but $x \notin\left[\widehat{x}_{B}^{U}, \widehat{x}_{A}^{U}\right]$. Obviously when condition (5) does not hold then $\Sigma_{I H}^{U}$ is empty and $\Sigma_{H}=\Sigma_{C H}^{U}$.

The next Proposition states our main result for $\Gamma_{U G}$. In order to derive this Proposition, we make use of both the sufficient condition developed by Ellison (2000) and some of the results for the NDG proved by Young (1993).

Proposition 3 Consider $\Gamma_{U G}$ and let Assumptions 1 and 2 be satisfied. When $\delta$ is sufficiently small the following cases are possible:

(a) if $V_{M} \leq \frac{V_{H}}{2}-c$ holds then $\Sigma_{s} \subseteq \Sigma_{I H}^{U}$ and a stochastically stable outcome exists. In this case all agents choose to invest and the surplus is equally split.

(b) if $V_{M}>\frac{V_{H}}{2}-c$ holds then no norm evolves.

Proof. See the Appendix

We now turn to the case in which a Dictator Game (instead of an Ultimatum Game) is played when an asymmetric investment profile is reached. In this case we denote by $x_{B}^{D}$ (resp. $V_{H}-x_{A}^{D}$ ) the share going to player $B$ (resp. player $A$ ) such that she receives an equilibrium payoff equal to $\delta$ when both agents have invested:

$$
\begin{aligned}
& x_{B}^{D}=c+\delta \\
& x_{A}^{D}=V_{H}-c-\delta .
\end{aligned}
$$

Since $c$ and $V_{H}$ are divisible by $\delta$, then $x_{B}^{D}, x_{A}^{D} \in D_{\delta}\left(V_{H}\right)$. Let

$$
\begin{aligned}
& \widehat{x}_{A}^{D}=\max \left\{x \in D_{\delta}\left(V_{H}\right) \mid\left(V_{H}-x\right) \frac{N-1}{N}-c \geq \delta\right\} \\
& \widehat{x}_{B}^{D}=\min \left\{x \in D_{\delta}\left(V_{H}\right) \mid x \frac{N-1}{N}-c \geq \delta\right\} .
\end{aligned}
$$

By keeping in mind Assumption 2 we obtain $\widehat{x}_{A}^{D}=x_{A}^{D}-\delta$ and $\widehat{x}_{B}^{D}=x_{B}^{D}+\delta$, i.e.

$$
\begin{aligned}
& \widehat{x}_{B}^{D}=c+2 \delta \\
& \widehat{x}_{A}^{D}=V_{H}-c-2 \delta .
\end{aligned}
$$

It is easy to see that $\widehat{x}_{B}^{D} \leq \widehat{x}_{A}^{D}$ provided:

$$
c<\frac{V_{H}-4 \delta}{2} .
$$

When this condition holds, we can define the following set

$$
\Sigma_{I H}^{D}=\left\{\theta \in \Sigma_{H} \mid x \in\left[\widehat{x}_{B}^{D}, \widehat{x}_{A}^{D}\right]\right\} .
$$


Also for $\Gamma_{D G}$ we can always partition the set $\Sigma_{H}$ into $\Sigma_{I H}^{D}$ and $\Sigma_{C H}^{D}$ where $\Sigma_{C H}^{D}=\Sigma_{H} \backslash \Sigma_{I H}^{D}$; the latter denotes the set of equilibria in which both agents choose to invest but $x \notin\left[\widehat{x}_{B}^{D}, \widehat{x}_{A}^{D}\right]$. However in this case the set $\Sigma_{I H}^{D}$ is always well-defined and $\Sigma_{C H}^{D}=\Sigma_{H} \backslash \Sigma_{I H}^{D}$ only supports $\left(x_{B}^{D}, x_{A}^{D}\right)$ as distributional rule. Indeed when $\delta$ is sufficiently small Point (b) of Assumption 1 ensures that cost $c$ is always smaller than $V_{H} / 2$. The following Proposition summarizes our finding concerning $\Gamma_{D G}$.

Proposition 4 Consider $\Gamma_{D G}$ and let Assumptions 1 and 2 be satisfied. When $\delta$ is sufficiently small (i.e. $\delta<\frac{V_{H}-2 c}{4}$ ), then $\Sigma_{s} \subseteq \Sigma_{I H}^{D}$ and a stochastically stable outcome always exists and it supports full investment and the egalitarian distributional rule.

Proof. See the Appendix

Proposition 4 states an intuitive result. Indeed in $\Gamma_{D G}$ the unique stochastically stable outcome is always $\left(H H, \frac{V_{H}}{2}, \frac{V_{H}}{2}\right)$ even when condition (5) does not hold. This implies that for whatever beliefs off the path (i.e. at $H L$ and/or $L H)$ the process still sustains the outcome $\left(H H, \frac{V_{H}}{2}, \frac{V_{H}}{2}\right)$. In particular, suppose that in period $t$ the process is at an efficient equilibrium $\theta$ in which the surplus is equally split but condition (5) does not hold. Let one $B$ agent expect to get almost the whole surplus if she does not invest. In period $t+1$ she will not invest. However, since at $H L$ all $A$ agents behave like a dictator, they will get almost the whole surplus. Hence in period $t+2$, when all $B$ agents revise, they all will choose to invest. Therefore the process does not leave the basin of attraction of $\theta$.

\section{Discussion}

In this Section, we provide further insights into our results. First consider $\Gamma_{U G}$. Borrowing from anthropological literature, we have suggested that this game is more apt for describing the evolution of social norms when property rights over the fruits of one's own independent investment are not socially supported. Proposition 3 describes the scenarios we can expect to observe over the long run in a society of this kind ${ }^{15}$. First, either a norm of investment and a norm of

\footnotetext{
15 Our results can be compared with Dawid and MacLeod (2001). If we put the assumptions $V_{L}=0$ and $V_{M}-c>0$ into their model, then the formal conditions for a single stochastically stable outcome stated in their Proposition 7 are in line with those stated in our Proposition 3. However their Proposition 7 is only concerned with the evolution of investment norms instead of the coevolution of investment and bargaining norms. As we said, this stems from deep differences between the two models and the evolutionary dynamics considered. In the preliminary version of the present paper we have also studied the model in which the surplus is equally split when both agents invest, as in Dawid and MacLeod (2001), but an Ultimatum Game occurs when only one agent has invested. In this case Proposition 3 continues to be true. Lastly Proposition 3 continues to hold even when in the UG the agent who makes a proposal is not the agent who has chosen to invest, as in Ellingsen and Robles (2002).
} 


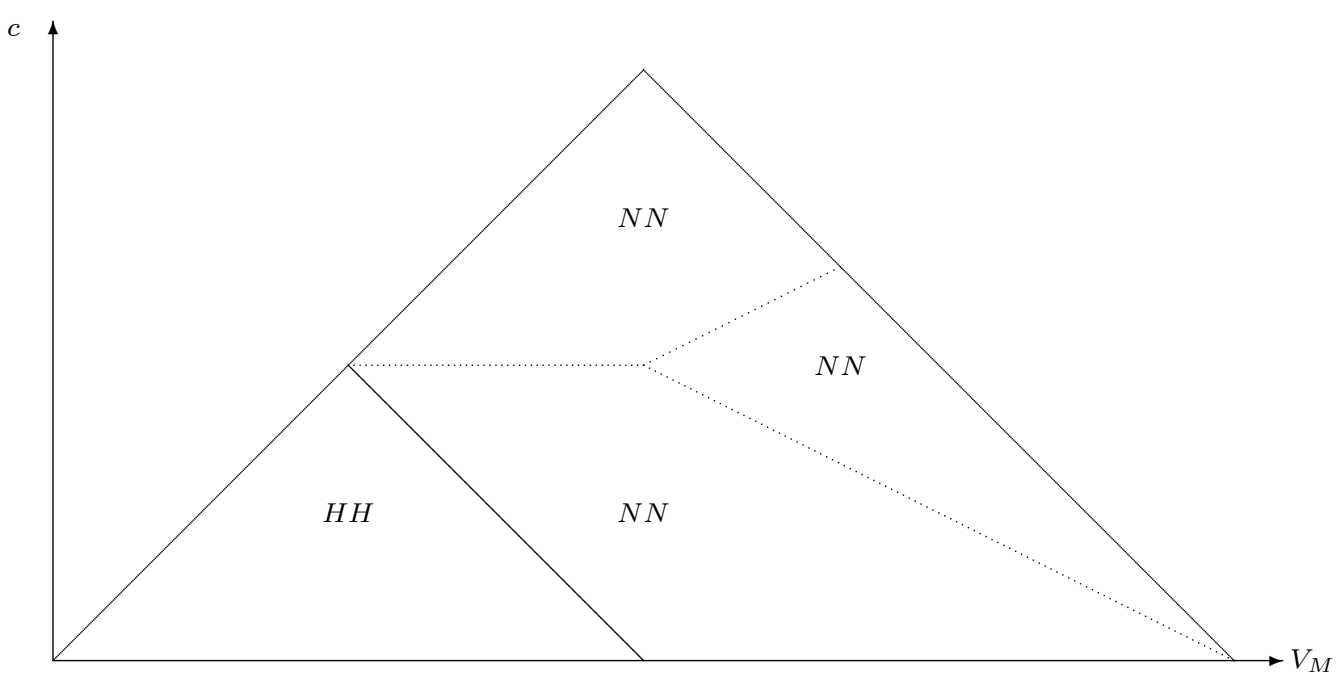

Fig. 1 Game $\Gamma_{U G}$. Region $H H$ denotes the parameter configuration supporting the unique stochastically stable outcome while region $N N$ denotes the parameter configuration in which no norms evolves since several stochastically stable outcomes can exist

bargaining coevolve, or no norm evolves at all. Second, when norms coevolve, they support efficient investment and egalitarian distribution.

According to Dawid and MacLeod (2008), investments are complements if the marginal effect of action $H$ when the opponent plays $H$ is greater than the marginal effect of action $H$ when the opponent plays $L$. Since in our model $V_{L}=0$, this condition is met when $V_{M}<\frac{1}{2} V_{H}$. Sure enough, when $\Sigma_{I H}^{U}$ is not empty investments are complements in the sense of Dawid and MacLeod (2001), but investments can be complements in the sense of Dawid and MacLeod also when $\Sigma_{I H}^{U}$ is not well defined. According to Proposition 3 we can thus expect norms to coevolve when investments are complements (i.e. $\left.V_{M}<\frac{1}{2} V_{H}\right)$ and the investment cost is sufficiently low $\left(c<\frac{V_{H}}{2}-V_{M}\right)$; these conditions are satisfied in region $H H$ of Figure 1, which is drawn under the assumption that $\delta$ is negligible. Instead, when investments are complements but the investment cost is higher than $\frac{V_{H}}{2}-V_{M}$, or when investments are not complements (i.e. $\frac{V_{H}}{2}<V_{M} \leq V_{H}$ ), we do not observe any norm; this happens in region $N N$ of Figure 1. In this region all investment profiles and any distributional rule can be observed in the long run.

To see this consider region $N N$ and suppose ${ }^{16}$ that the only outcome observed is $\left(H H, \frac{V_{H}}{2}, \frac{V_{H}}{2}\right)$. Since no high-low matches are observed, the beliefs on bargaining outcomes in high-low matches can drift. Because of said drift, all agents $B$ might, for instance, deem that $A$ will only accept a distribution granting her almost the whole pie at $L H$ profile even if all $B$ are ready to accept any proposal at $H L$. Sure enough the drift does not shift the process from the equilibrium outcome $\left(H H, \frac{V_{H}}{2}, \frac{V_{H}}{2}\right)$. Suppose now that one agent $A$

16 We remind that in region $N N$ condition (5) does not hold. 


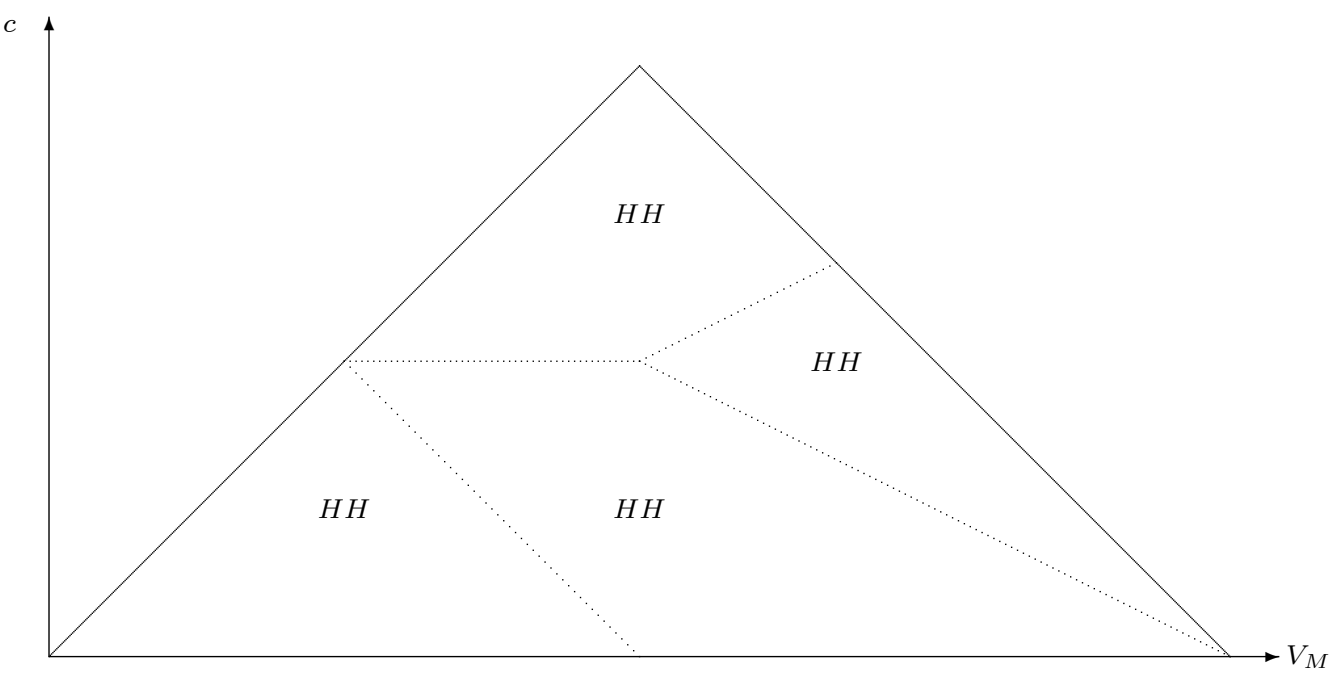

Fig. 2 Game $\Gamma_{D G}$. Region $H H$ denotes the parameter configuration supporting the unique stochastically stable outcome

deems to capture a share $V_{M}-\delta$ at $H L$. According to this new belief she will play $L$ in the next period. Therefore the initial equilibrium $\left(H H, \frac{V_{H}}{2}, \frac{V_{H}}{2}\right)$ can be overturned when both populations update, once at the time, so that the process enters the basin of attraction of $(L L, 0,0)$ eventually. However this cannot be a social norm since a sequence of single mutations is enough to move the system into the basin of attraction of another equilibrium supporting a single outcome with a different investment profile (see Lemma 2, point (a), and Lemma 3). And so on and so forth.

We have also seen that $\Gamma_{D G}$ seems more appropriate for describing the evolution of social norms when property rights over the fruits of one's own independent investment are socially protected. In this context, Proposition 4 ensures that norms always coevolve. This result is illustrated in Figure 2, in which region $N N$ of the previous Figure 1 disappears and is replaced by region $H H$. Therefore, region $H H$ now coincides with the whole parameter space compatible with Assumption 1. Indeed, since in this case the only agent who has invested behaves as a dictator, any drift of beliefs does not upset the outcome $\left(H H, \frac{V_{H}}{2}, \frac{V_{H}}{2}\right)$ even when condition (5) is not satisfied. Confidence in the dictator's unselfish behavior is thus always shortsighted.

In both games, when social norms emerge, they support the efficient investment profile and the egalitarian distribution of the surplus. The fact that the distributional norm is egalitarian is not a surprise since our model is symmetric. In turn this symmetry depends on the assumptions that investment costs are identical and that the surplus accrued when only one agent invests does not depend on the identity of the investing agent. One may then reasonably question whether an egalitarian norm could still be expected to emerge when some form of heterogeneity is allowed (Baker and Swope 2005). This is 
an important point since the literature on modern hunters-gatherers suggests that, in some cases, these societies endorse an egalitarian rule of distribution even when differences in hunting-gathering abilities are observed (Kaplan and Hill, 1985; Woodburn, 1982) while in some other cases they do not (Hawkes 1992). This point is developed in a companion paper.

Our results are derived under the natural assumption that full cooperation in the production stage is efficient. We now briefly provide some insights on its role; this allows us to evaluate a conjecture recently advanced by Alvard (2004). In discussing the results of some experiments in fifteen small scale societies ${ }^{17}$, Alvard (2004) suggested that norms of cooperation and norms of fair division were likely to be observed in societies in which "the payoffs to cooperation are high". Hence by relaxing our efficiency assumption and by comparing the results we can test the cutting power of Alvard's hypothesis in our settings. In order to do this we keep all the assumptions so far made with the exception of point (b) of Assumption 1 which is replaced by either $V_{M}-c>V_{H}-2 c$ or $V_{M}-c<V_{H}-2 c$. Under this new scenario the parameter region enlarges by embracing the triangle on the right hand side of the previous parameter space (see Figure 3). In this appended area all our intermediate results are still valid. However condition (5) can no longer be satisfied; as a consequence only point (b) of Proposition 3 holds. Therefore in $\Gamma_{U G}$ no norm evolves in the added region due to the failure of said condition; consequently region $N N$ enlarges. On the other side, in $\Gamma_{D G}$ Proposition 4 continues to be true so that the same social norms coevolve with and without the efficiency hypothesis.

Lastly, we speculate on the role played by bargaining in the evolution of social norms in $\Gamma_{U G}$ and in $\Gamma_{D G}$ under the efficiency assumption. In order to do this, we apply the evolutionary framework to a simpler model in which a distributional norm already exists and agents only have to coordinate their investment decisions. To this end we consider the one shot game suggested by Hawkes (1992, payoff matrix 8 ). Let $\Gamma_{C}$ denote this model. The resulting game is represented by the normal form (12) in which we assume ${ }^{18}$ (i) that for whatever investment profile the pie is equally split $^{19}$; (ii) that the net surplus arising when both agents invest is the greatest one, and (iii) that the net surplus accruing when only one invests is not negative. The latter two conditions are satisfied when $c<\min \left(\frac{1}{2} V_{H}, V_{H}-V_{M}, V_{M}\right)$. In this strategic framework, when there is more than one pure Nash equilibrium, the stochastically stable one coincides with the risk dominant equilibrium (Young 1993a). From an anthropological perspective, this game is suitable to describe a situation arising when the pie to be distributed has to be produced in advance but, since

\footnotetext{
17 See Heinrich et al. (2004). An assessment of these experiments can be found in Chibnik (2005) and in Hagen and Hammerstein (2006).

18 This game tallies with Hawkes's game under the assumptions $V=V_{H}$ and $s V=V_{M}$.

19 This means that the distributional rule in $\Gamma_{C}$ coincides with the unique distributional norm which can evolve in $\Gamma_{U G}$.
} 

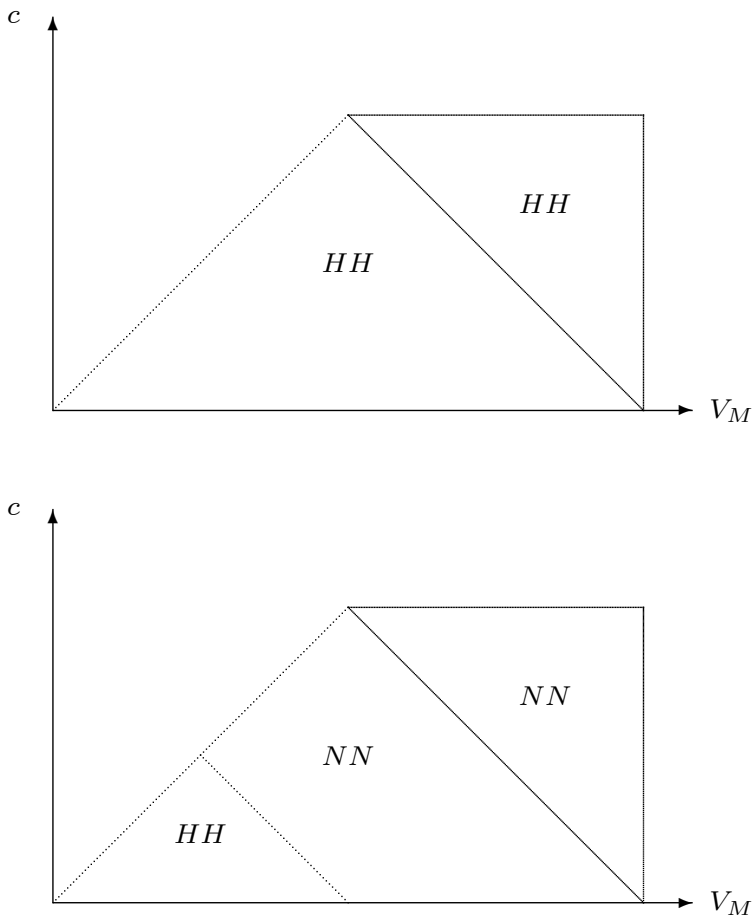

Fig. 3 Top: game $\Gamma_{D G}$; bottom: game $\Gamma_{U G}$. In both figures the triangle on the right hand side denotes the parameter configuration for which investment by both agents is not efficient. No norms are observed in $\Gamma_{U G}$ while norms co-evolve in $\Gamma_{D G}$

property rights cannot be enforced ${ }^{20}$, it must always be split equally.

$$
\begin{array}{ccc}
\multicolumn{1}{c}{} & H & L \\
\cline { 2 - 3 } H & \frac{V_{H}}{2}-c, \frac{V_{H}}{2}-c & \frac{V_{M}}{2}-c, \frac{V_{M}}{2} \\
L & \frac{V_{M}}{2}, \frac{V_{M}}{2}-c & 0,0
\end{array}
$$

Few computations show that the game has either three Nash equilibria (two in pure strategies and one in mixed strategies) or only one pure symmetric Nash equilibrium. When the game admits a unique Nash equilibrium, this is investment profile $H H$ if $c<\min \left(\frac{V_{M}}{2}, \frac{V_{H}-V_{M}}{2}\right)$ and investment profile $L L$ if $c>\max \left(\frac{V_{M}}{2}, \frac{V_{H}-V_{M}}{2}\right)$. When the game admits two pure Nash equilibria, these are $H H$ and $L L$ if $\frac{V_{M}}{2}<c<\frac{V_{H}-V_{M}}{2}$ and $H L$ and $L H$ if $\frac{V_{H}-V_{M}}{2}<c<\frac{V_{M}}{2}$.

20 According to the anthropologists, this situation is compatible with societies admitting the so-called tolerated theft (Hawkes 1992). This means that sharing also occurs when the pie is only provided by one agent, since excluding outsiders is too costly. However, Bell (1995) argued that tolerated theft presumes that society ensures the hunter the full right to his or her catch, a condition that may not be granted. 


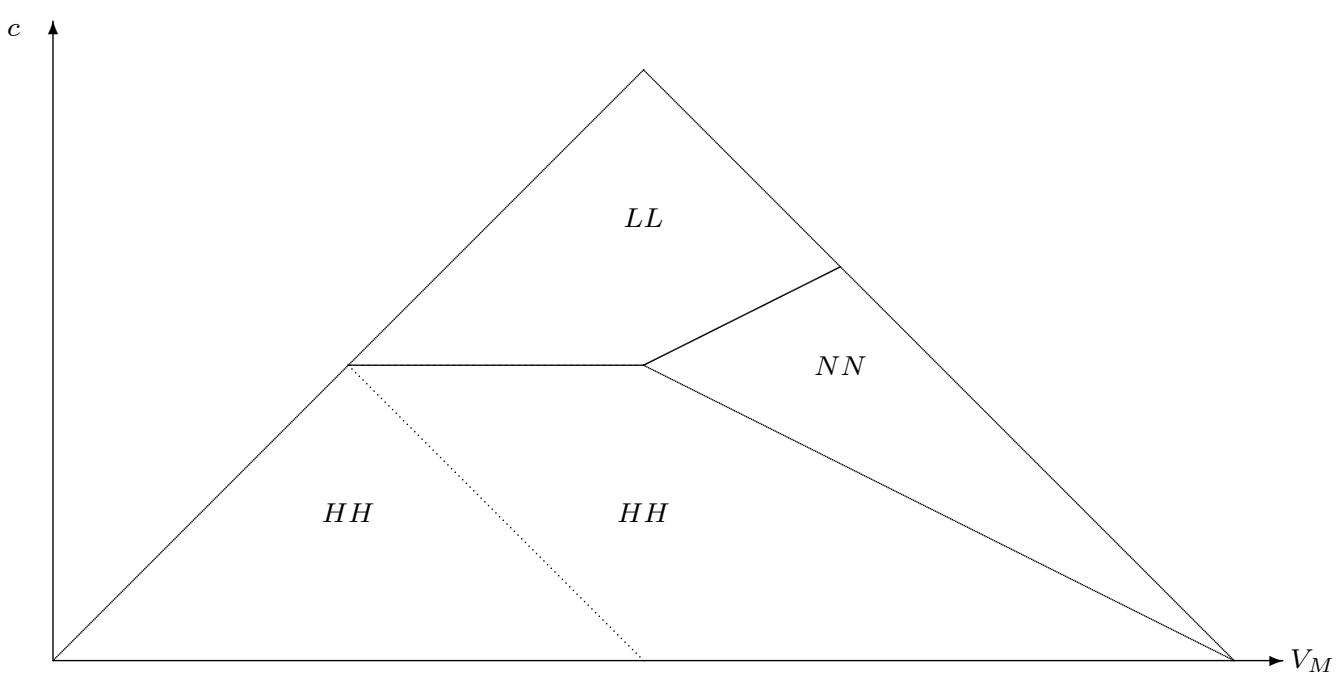

Fig. 4 Game $\Gamma_{C}$. Parameter configuration in which efficient investment $(H H)$ and no investment $(L L)$ are stochastically stable, compared with the parameter configuration $(N N)$ where no investment norm evolves

When the game has two pure symmetric equilibria, the stochastic stability selects one of them. In particular, it selects investment profile $H H$ if $\frac{V_{M}}{2}<c<$ $\min \left(\frac{V_{H}-V_{M}}{2}, \frac{V_{H}}{4}\right)$, while it selects investment profile $L L$ if $\max \left(\frac{V_{M}}{2}, \frac{V_{H}}{4}\right)<$ $c<\frac{V_{H}-V_{M}}{2}$. When instead the game has two pure asymmetric equilibria, $L H$ and $H L$, both are stochastically stable.

These results are illustrated in Figure 4, where $H H$ (resp. $L L$ ) denotes the region in which investment profile $H H$ (resp. $L L$ ) is the only stochastically stable equilibrium and where $N N$ denotes the region in which no investment norms emerge since both equilibria, $L H$ and $H L$, are stochastically stable. In this last game, a coordination failure arises when investment profile $L L$ is the only stochastically stable equilibrium. Direct comparison of Figures $1\left(\Gamma_{U G}\right)$ and 4 allows us to argue that the main consequences of modeling a bargaining stage as a game is that the region supporting the worst outcome $(L L)$ disappears while the region supporting the best outcome $(H H)$ shrinks. Hence, the region in which no investment and bargaining norms evolve is enlarged. This highlights the fact that, contrary to what happens in $\Gamma_{C}$ in which an egalitarian distributional norm is already established, when a distribution norm is allowed to evolve, its evolutionary process affects the evolution of the investment norm (Hackett 1993, 1994). When we do the same exercise for $\Gamma_{D G}$, we obtain a new game $\Gamma_{C}^{\prime}$ which differs from $\Gamma_{C}$. This is because at the asymmetric investment profiles, the whole surplus goes to the investing agent only. Under the structural assumptions of the paper, it turns out that $H$ is now a dominant strategy and $H H$ is the only Nash equilibrium. ${ }^{21}$ Since $\Gamma_{C}$ and $\Gamma_{C}^{\prime}$

21 We remark that in $\Gamma_{C}^{\prime}$ any distributional norms granting a positive payoff when both invest does not hamper the evolution of the investment norm. 
essentially differ in the degree of property protection assured, we conclude that modeling a bargaining stage is crucial for understanding the evolution of both investment and distribution norms in societies in which full rights of possession are not socially supported. In societies in which full rights of possession are socially protected, this only explains which bargaining norm is brought to bear.

\section{A Appendix}

First of all we introduce some useful notations. We denote by $\lfloor s\rceil$ the least integer number greater than $s$ when $s$ is not an integer and $(s+1)$ is otherwise. Consider a state $\theta$ and suppose that all agents observe $z(\theta)$. Consider $\Gamma_{U G}$; for an agent $i \in A$, action $L$ is not preferred to action $H$ if;

$$
p_{B}(\theta)\left(\widetilde{y}_{H H}^{i}(\theta)-\widetilde{y}_{L H}^{i}(\theta)-c\right)+\left(1-p_{B}(\theta)\right)\left(\widetilde{y}_{H L}^{i}(\theta)-c\right) \geq 0
$$

analogously, action $L$ is not preferred to action $H$ for agents $i \in B$ if

$$
p_{A}(\theta)\left(\widetilde{x}_{H H}^{i}(\theta)-\widetilde{x}_{H L}^{i}(\theta)-c\right)+\left(1-p_{A}(\theta)\right)\left(\widetilde{x}_{L H}^{i}(\theta)-c\right) \geq 0 .
$$

Here, by $p_{A}(\theta)$ (resp. $p_{B}(\theta)$ ) we denote the frequency of agent $A$ (resp. $B$ ) who played $H$ in $\theta$, and by $\widetilde{y}_{H H}^{i}(\theta)$ (resp. $\widetilde{x}_{H H}^{i}(\theta)$ ) the expected payoffs of agent $i \in A$ (resp. $i \in B$ ) at the information set $H H$, given $z(\theta)$. Similar conditions hold for $\Gamma_{D G}$

From now on, by slightly abusing notation, $\left(H H, y_{H H}, x_{H H}\right)$ denotes a terminal node in which both agents have chosen $H$, agent $A$ makes a demand $y_{H H}$ and agent $B$ makes a demand $x_{H H}$. This applies for the other terminal nodes, too.

The following results will be used afterwards.

Lemma 4 Let $x_{H H, 1}<x_{H H, 2}<\ldots<x_{H H, k}$ be the demands made by $B$ at $H H$ for some state $\theta$. Then the set of best behavioral demands following $H H$ for agents $A$ is a subset of $\left\{V_{H}-x_{H H, l}\right\}_{l=1}^{k}$.

Proof. See Lemma A.1 in Ellingsen and Robles (2002).

Lemma 5 Consider $\Gamma_{U G}$ and let $\Omega$ be a limit set of $(\Theta, P)$. If $\left(H L, y_{H L}, x_{H L}\right) \in \rho(\Omega)$ $\left[\right.$ resp. $\left.\left(L H, y_{L H}, x_{L H}\right) \in \rho(\Omega)\right]$ then:

(i) $x_{H L}=V_{M}-y_{H L}\left[\right.$ resp. $\left.y_{L H}=V_{M}-x_{L H}\right]$

(ii) $\left(H L, y_{H L}, x_{H L}\right)\left[\right.$ resp. $\left.\left(L H, y_{L H}, x_{L H}\right)\right]$ is the only outcome which supports investment profile $H L[$ resp. $L H]$ in $\rho(\Omega)$.

Proof. We only consider profile $H L$. The same holds true for $L H$.

Point (i). Let $\theta$ be a state such that: (a) $\theta \in \Omega$; (b) $\left(H L, y_{H L}, x_{H L}\right)$ belongs to the support of $z(\theta)$ and $x_{H L} \neq V_{M}-y_{H L}$. Let us suppose that only $B$ agents update their characteristics: they will all accept $y_{H L}$. For whatever belief on opponent's behavior this action is always a best reply. It is then impossible to return to the original state $\theta$. This contradicts the assumption that $\theta \in \Omega$.

Point (ii). First we show that $\Omega$ cannot include a state $\theta$ in which multiple demands are made at $H L$. Subsequently, we show that $\Omega$ cannot include two different states supporting different outcomes following $H L$.

Let $\theta$ be a state such that: (a) $\theta \in \Omega$ and (b) multiple demands are made by agents $A$ at $H L$. We already know from point (i) that at $\theta$ all agents $B$ accept all the demands made by their opponents. Suppose only agents $A$ revise their characteristics; then any agent $A$ 
will make the maximum demand observed at $H L$. Hence, it is impossible to return to the original state $\theta$. This contradicts the assumption that $\theta \in \Omega$.

Now let $\theta$ and $\theta^{\prime}$ be two states such that: (a) both states belong to $\Omega$ and (b) $H L$ is observed. A single demand is made by $A$ but $y_{H L}\left(\theta^{\prime}\right)>y_{H L}(\theta)$. Since it is impossible to return to $\theta$ then assumption $\theta \in \Omega$ is contradicted.

Lemma 6 Let $\Omega$ be a limit set of $(\Theta, P)$. If $\left\{(H H, y, x) ;\left(H H, y^{\prime}, x^{\prime}\right)\right\} \in \rho(\Omega)$ and either $x \neq x^{\prime}$ or $y \neq y$, then $\Omega$ is a singleton and a self-confirming equilibrium of $\Gamma$.

Proof. Consider a set $\Omega$ and let $\theta \in \Omega$ be a state in which at least two demands have been observed in one population (i.e. $B$ ). Suppose that at least one of these demands $\left(x^{*}\right)$ is not a best reply to $z(\theta)$. Suppose also that, after observing $z(\theta)$, all agents who demanded $x^{*}$ revise; as a consequence $x^{*}$ disappears. A new state $\bar{\theta} \in \Omega$ is then reached in which profile $H H$ is still observed. Suppose now that all $A$ update; then, by Lemma 4, nobody will make demand $\left\{V_{H}-x^{*}\right\}$. These two demands have thus disappeared and it is impossible to return to the original state $\theta$. This contradicts the assumption that $\theta \in \Omega$. Therefore, if multiple demands are made, each must be a best reply to $z(\theta)$.

Now consider an agent belonging to population $A$ who has played $H$ in $\theta$ and suppose this agent has the incentive to change her investment should she know $z(\theta)$. When this agent updates, the distribution of the demands made by population $A$ in subgame $H H$ differs from the original. This implies that at least one demand made by some opponents (i.e. $B$ ) is no longer a best reply when $B$ updates. By applying the argument made in the paragraph above, we conclude that at least one pair of demands has disappeared and cannot reappear. This contradicts the assumption that $\theta \in \Omega$.

By Lemma 5 since the set $\rho(\Omega)$ can include at most one outcome following the profile $H L$ or $L H$ then state $\theta$ must be a self-confirming equilibrium.

Proof of Proposition 1. Assume that $\Omega$ is not a singleton. We know from Lemmas 5 and 6 that, if a bargaining subgame is reached, only one of its terminal nodes is observed. First we show that $\rho(\Omega)$ must contain one outcome for every bargaining subgame. Of course $\rho(\Omega)$ must differ from $\left\{\left(H H, y_{H H}, V_{H}-y_{H H}\right),(L L, 0,0)\right\}$. Suppose $\rho(\Omega)$ includes the following outcomes: (a) $\left(H H, y_{H H}, x_{H H}\right)$ with $y_{H}+x_{H H}=V_{H}$; (b) $\left(H L, y_{H L}, x_{H L}\right)$ with $y_{H L}+x_{H L}=V_{M}$. In $\Omega$ a state $\theta$ in which both outcomes are observed must exist and it cannot be an equilibrium. We show that from $\theta$ it is possible to reach either the basin of attraction of one equilibrium or a state in which all bargaining nodes are observed. Suppose some agents $B$ update. If $x_{H H}-c>x_{H L}$ then they will choose $H$ so that, at the new state $\theta^{\prime}$, the frequency of this action in population $B$ will increase. Suppose now that at least one agent $A$ has beliefs $\widetilde{y}_{L H}^{i}$ leading her not to prefer $H$ to $L$ when all agents $B$ play $H$; then, starting from $\theta$, it is possible to reach a state in which all investment profiles are realized. To see this suppose $y_{H H}-\widetilde{y}_{L H}^{*}-c<0$ but $y_{H L}-c>0$. Let $p_{B}^{i^{*}}$ be:

$$
p_{B}^{i^{*}}=\left\lfloor\frac{c-y_{H L}}{\left(y_{H H}-\widetilde{y}_{L H}^{i^{*}}-c\right)}\right\rceil .
$$

Consider now the case in which $p_{B}^{i^{*}}$ agents $B$ have revised at $\theta$ and only agent $i^{*} \in A$ observes the distribution of outcomes $z\left(\theta^{\prime}\right)$. Since the specific agent $i^{*} \in A$ will play $L$, then all investment profiles are realized afterwards. Therefore this contradicts the assumption that $\rho(\Omega)=\left\{\left(H H, y_{H H}, x_{H H}\right) ;\left(H L, y_{H L}, x_{H L}\right)\right\}$. Otherwise, by letting all agents $B$ update from $\theta$ it is likely to reach the basin of attraction of one equilibrium of the game supporting the outcome $\left(H H, y_{H H}, x_{H H}\right)$. If $x_{H H}-c \leq x_{H L}$ we reach the same conclusion by using a similar argument. It is simple to see that the same conclusion holds when $\rho(\Omega)$ includes any two different outcomes. Therefore if $\Omega$ is a not a singleton, all the bargaining nodes are visited meaning that $\rho(\Omega)$ includes four outcomes each of which is a subgame equilibrium.

We now have to show that the payoffs must satisfy a well-defined set of constraints. Notice that a state $\theta \in \Omega$ in which all the investment profiles are observed must exist. Moreover when we allow all agents to update, all agents $A$ will choose $H$ :

$$
p_{B}(\theta)\left(y_{H H}-y_{L H}-c\right)+\left(1-p_{B}(\theta)\right)\left(y_{H L}-c\right)>0,
$$


and all agents $B$ will choose $H$ :

$$
p_{A}(\theta)\left(x_{H H}-x_{H L}-c\right)+\left(1-p_{A}(\theta)\right)\left(x_{L H}-c\right)>0 .
$$

We can rewrite these conditions as

$$
\begin{aligned}
& p_{B}(\theta) A_{1}+\left(1-p_{B}(\theta)\right) A_{2}>0 \\
& p_{A}(\theta) B_{1}+\left(1-p_{A}(\theta)\right) B_{2}>0 .
\end{aligned}
$$

First of all notice that all $A_{i}$ and $B_{i}$ can not be null since this would imply that $\theta$ is an equilibrium and $\Omega$ a singleton. Furthermore, when - for some populations - both expressions are either not negative or not positive, and at least one is not null, then the process can reach a new state from $\theta$ which is a self-confirming equilibrium. Consider the case in which both expressions are null for population $A$ only. When $B_{1}$ is strictly positive and $B_{2}$ is strictly negative all $B s$ prefer $H$ if $p_{A}(\theta)>p_{A}^{*}$ where:

$$
p_{A}^{*}=\frac{c-x_{L H}}{\left(x_{H H}-x_{H L}\right)-x_{L H}} .
$$

Otherwise when $B_{1}$ is strictly negative and $B_{2}$ is strictly positive all $B s$ prefer $H$ if $p_{A}(\theta)<$ $p_{A}^{*}$. In both cases, when all $B$ agents update they will choose $H$. Hence a state which is an equilibrium of the game can be reached from $\theta$. When both expressions $B_{1}$ and $B_{2}$ are null, we get a similar conclusion where the threshold value of $p_{B}(\theta)$ is:

$$
p_{B}^{*}=\frac{c-y_{H L}}{\left(y_{H H}-y_{L H}\right)-y_{H L}} .
$$

We are left with the case in which the product of the corresponding two expressions is strictly negative for each population. However, when $A_{1}$ and $B_{1}$ have the same sign a similar argument allows us to reach the same conclusion. Indeed, suppose that both $A_{1}$ and $B_{1}$ are strictly positive. This implies that all $B s$ prefer $H$ if $p_{A}(\theta)>p_{A}^{*}$ and all $A s$ prefer $H$ if $p_{B}(\theta)>p_{B}^{*}$. Hence, for whatever values of $p_{A}(\theta)$ and $p_{B}(\theta)$, starting from $\theta$ the process can reach an equilibrium when one population revises at a time. The remaining possible case occurs when $B_{1} B_{2}<0$ and $A_{1} A_{2}<0$ but $A_{1} B_{1}<0$.

Proof of Proposition 2. It follows by applying the same arguments used in the Proof of Proposition 1 and taking into account that $y_{H L}=x_{L H}=V_{M}-\delta$ holds at any limit set. In this case the conditions $B_{1} B_{2}<0, A_{1} A_{2}<0$ and $A_{1} B_{1}<0$ can not be simultaneously met because both $A_{2}$ and $B_{2}$ are strictly positive. Consequently all limit sets are singleton. In addition an equilibrium of the game can only support outcome $(L L, 0,0)$ if it also supports at least one outcome following each investment profile.

Before giving the proof we briefly review the concept of mutation connected set.

Definition 1 Consider a union of limit sets $X$. This set is mutation connected if for all pairs $\Omega, \Omega^{\prime} \in X$ exists a sequence of limit sets $\left(\Omega_{1}=\Omega, \Omega_{2}, \ldots, \Omega_{n}=\Omega^{\prime}\right)$ such that (a) for any $k \in\{1, \ldots, n-1\}, \Omega_{k} \in X$ and (b) every transition from $\Omega_{k}$ to $\Omega_{k+1}$ needs no more than one mutation.

Consider a limit set $\Omega$ which does not support all information sets and suppose a single mutation occurs. If this mutation is a drift then the process reaches a new limit set $\Omega^{\prime}$ which differs from $\Omega$ only for some belief and/or action at some unreached information sets. Let $\Sigma(\Omega)$ be the set of equilibria which only differ from $\Omega$ for some beliefs (and/or actions) held in some unreached information set. Sure enough the set $\Sigma(\Omega)$ is mutation connected. When $\Omega$ is singleton, namely $\Omega=\{\theta\}$, we use $\Sigma(\theta)$ instead of $\Sigma(\Omega)$.

Proof of Lemma 1. We give the detailed proof for $\Gamma_{U G}$; we then suggest how to adapt it to $\Gamma_{D G}$. When multiple demands are observed at $H H$, we denote by $\left\{x_{H H, l}\right\}_{l=1}^{k}$ the ordered 
sets of demands made by $B$ and by $\left\{y_{H H, l}\right\}_{l=1}^{k}$ the ordered sets of demands made by $A$. By iterative applications of Lemma (4) we get $\left\{y_{H H, l}\right\}_{l=1}^{k}=\left\{V_{H}-x_{H H, l}\right\}_{l=k}^{1}$. Since $\theta$ is an equilibrium then the expected payoff at $H H$ are:

$$
\begin{aligned}
& \widetilde{y}_{H H}^{i}(\theta)=y_{H H, 1}=V_{H}-x_{H H, k} ; \forall i \in A \\
& \widetilde{x}_{H H}^{i}(\theta)=x_{H H, 1}=V_{H}-y_{H H, k} ; \forall i \in B
\end{aligned}
$$

where $y_{H H, 1}=y_{H H, k} \eta_{1}^{B}$ and $x_{H H, 1}=x_{H H, k} \eta_{1}^{A}$ and $\eta_{1}^{B}$ (resp. $\eta_{1}^{A}$ ) is the fraction of $B$ (resp. A) who claim $x_{H H, 1}\left(\right.$ resp. $\left.y_{H H, 1}\right)$ under $\theta$.

I) Consider an equilibrium $\theta$ in which only the investment profile $H H$ is observed and multiple demands are made. Let a single agent $B$ switch from $x_{H H, k}$ to $x_{H H, 1}$. When agents $A$ update they will make a demand $y_{H H, k}=V_{H}-x_{H H, 1}$. Hence, we reach a new equilibrium $\theta^{\prime}$ in which only $H H$ is observed and only the two demands $\left(V_{H}-x_{H H, 1}, x_{H H, 1}\right)$ occur.

II) Suppose now that two profiles are observed at the equilibrium $\theta$. We give the proof only when $H H$ and $H L$ are observed. The other remaining cases are similar.

II.1) Consider the case in which multiple demands are made following $H H$. Since $\theta$ is an equilibrium, the following conditions must always be met:

$$
\begin{aligned}
& p_{B}(\theta)\left(y_{H H, 1}-\widetilde{y}_{L H}^{i}(\theta)-c\right)+\left(1-p_{B}(\theta)\right)\left(y_{H L}-c\right) \geq 0, \forall i \in A \\
& \left(V_{H}-y_{H H, k}\right)-c=V_{M}-y_{H L}, \forall i \in B .
\end{aligned}
$$

When $y_{H H, 1}-c>\delta$, by a sequence of single mutations the process can get from $\theta$ to $\theta_{1} \in \Sigma(\theta)$ where $y_{H H, 1}-\widetilde{y}_{L H}^{i}\left(\theta_{1}\right)-c>0$ for all $A s$. At $\theta_{1}$ let a single agent $A$ mutate from $y_{H H, k}$ to $y_{H H, 1}$ and let all agents $B$ revise; as a consequence they will all choose $H$ and ask $\left(V_{H}-y_{H H, 1}\right)$. Therefore, the process reaches a new equilibrium $\theta^{\prime}$ where $\rho\left(\theta^{\prime}\right)=$ $\left\{H H, y_{H H, 1}, V_{H}-y_{H H, 1}\right\}$. When instead $y_{H H, 1}-c \leq \delta$, the inequality $y_{H L}-c \geq 0$ must hold for all $A$ s. Suppose a single $A$ mutates from $y_{H H, k}$ to $\bar{y}$ where $\bar{y}>y_{H H, k}$ and let all agents $B$ update: as a consequence they all will choose $L$. Therefore, by a sequence of single mutations the process reaches a new equilibrium $\theta^{\prime}$ where $\rho\left(\theta^{\prime}\right)=\left\{H L, y_{H L}, V_{M}-y_{H L}\right\}$.

II.2) Now consider the case in which a single demand is made following $H H$. Suppose $y_{H L}-c \geq 0$, the process can reach a new equilibrium $\theta^{\prime}$ where $\rho\left(\theta^{\prime}\right)=\left\{H L, y_{H L}, V_{M}-y_{H L}\right\}$ when a single agent $A$ mutates from $y_{H H, 1}$ to $\bar{y}$ where $\bar{y}>y_{H H, 1}$ and all $B s$ revise. Suppose instead $y_{H L}-c<0$, then: (a) Point (c) of Assumption 1 implies that the subgame $\left(H L, V_{M}-\delta\right)$ at $\theta$ is not reached; (b) $y_{H H, 1}-\widetilde{y}_{L H}^{i}\left(\theta_{1}\right)-c \geq 0$ for every $A$. By drifting, all agents $B$ are led to accept the maximum feasible demand made by $A$ in $H L$ so that a new equilibrium $\theta_{1}$ is reached. Sure enough, $\theta_{1} \in \Sigma(\theta)$. Suppose now that a single agent $A$ changes her demand from $y_{H L}$ to $V_{M}-\delta$. When all agents $A$ update, they observe that all $B s$ have accepted the demand $V_{M}-\delta$; therefore, in $H L$ their best response is $y_{H L}=V_{M}-\delta$. When all agents $B$ update, they will choose $H$ being $x_{H L}=\delta$. Hence, the process reaches equilibrium $\theta^{\prime}$ where $\rho\left(\theta^{\prime}\right)=\left\{H H, y_{H H, 1}, V_{H}-y_{H, 1}\right\}$.

III) Suppose now that all investment profiles are observed at equilibrium $\theta$. Since $\theta$ is an equilibrium the following conditions must be satisfied:

$$
\begin{aligned}
& p_{B}(\theta)\left(y_{H H, 1}-y_{L H}-c\right)+\left(1-p_{B}(\theta)\right)\left(y_{H L}-c\right)=0 \\
& p_{A}(\theta)\left(x_{H H, 1}-x_{H L}-c\right)+\left(1-p_{A}(\theta)\right)\left(x_{L H}-c\right)=0 .
\end{aligned}
$$

where $y_{H H, 1}=V_{H}-x_{H H, k}, y_{H L}=V_{M}-x_{H L}$ and $y_{L H}=V_{M}-x_{L H}$. We may rewrite these conditions as

$$
\begin{aligned}
& p_{B}(\theta) A_{1}^{\prime}+\left(1-p_{B}(\theta)\right) A_{2}^{\prime}=0 \\
& p_{A}(\theta) B_{1}^{\prime}+\left(1-p_{A}(\theta)\right) B_{2}^{\prime}=0 .
\end{aligned}
$$

We argue that when the second expression $\left(A_{2}^{\prime}\right.$ or $\left.B_{2}^{\prime}\right)$ is not positive for at least one population then the process, through a sequence of single-mutations, can reach one equilibrium supporting a smaller number of investment profiles. In order to see this suppose, for instance, that $A_{2}^{\prime}<0$; in this case Point (c) of Assumption 1 assures that the subgame 
$\left(H L, V_{M}-\delta\right)$ is not reached at $\theta$. A drift can lead all agents $B$ to accept the opponent's maximum feasible demand at $H L$. A new $\theta_{1} \in \Sigma(\theta)$ is then reached. Suppose now that at this new equilibrium a single agent $A$ mutates her demand from $y_{H L}$ to $V_{M}-\delta$. When all agents $A$ revise, they will play $H$ and will make a demand $y_{H L}=V_{M}-\delta$. Now let all agents $B$ update. Since each agent $B$ knows that $x_{H L}=\delta$ and that all $A s$ have played $H$, then her best reply depends on the sign of $\left(x_{H H, 1}-\delta-c\right)$. However, it is simple to see that for any value of $\left(x_{H H, 1}-\delta-c\right)$, the process can reach a new equilibrium in which a smaller number of investment profiles is realized. If, at this new equilibrium, two investment profiles are realized, then the process can reach an equilibrium which supports a single outcome by a further sequence of single transition (see point II.2 above). When both $A_{2}^{\prime}$ and $B_{2}^{\prime}$ are positive, a single mutation occurring in population $A$ is enough to move the process from $\theta$ to a new equilibrium $\theta^{\prime}$ where $\rho\left(\theta^{\prime}\right)=\left\{L H, y_{L H}, V_{M}-y_{L H}\right\}$. The mutation needed depends on how many demands are observed at $H H$. In particular: (i) when multiple demands are made at $H H$, one mutation from $y_{H H, k}$ to $y_{H H, 1}$ is enough; (ii) when only one demand is made at $H H$, one mutation from $H$ to $L$ is enough.

IV) The remaining case occurs when $\Omega$ is not a singleton. Under the Point (c) of Assumption 1 at least one of the following two subgames $\left(L H, V_{M}-\delta\right)$ and $\left(H L, V_{M}-\delta\right)$ is never reached. The same argument used above implies that the population can get from $\Omega$ to $\theta^{\prime}$ through a sequence of single-mutations.

Now consider game $\Gamma_{D G}$. The above-mentioned arguments continue to work with minor modifications. In particular since $y_{H L}=x_{L H}=V_{M}-\delta$ then: (a) in case II) the set of investments profiles supported by an equilibrium can either be $\{H H, H L\}$ or $\{H H, L H\}$; (b) in case III) both $A_{2}^{\prime}$ and $B_{2}^{\prime}$ are positive; (c) case IV) does not arise.

Proof of Lemma 2. Since in $\Gamma_{D G}$ the set $\Sigma_{L}$ is empty, the first point of the lemma holds for $\Gamma_{U G}$ only.

(a) Let $\theta$ be an equilibrium belonging to $\Sigma_{L}$. From $\theta$, by a sequence of single mutations, the process reaches a new equilibrium $\theta^{*} \in \Sigma(\theta)$ in which for every agent $A$ and $B$ it is true that: (i) $\widetilde{y}_{H H}^{i}\left(\theta^{*}\right)=V_{H}-x_{H H}$ and $V_{H}-x_{H H}>c+\delta$; (ii) at the subgame $\left(L H, V_{M}-\delta\right.$ ) each agent $A$ accepts (i.e. she chooses $\delta$ ); (iii) $\widetilde{x}_{H L}^{i}\left(\theta^{*}\right)=\delta$ and $\widetilde{x}_{H H}^{i}\left(\theta^{*}\right)=x_{H H}$ and $x_{H H}-\delta-c \geq 0$. Suppose now an agent $B$ mutates by playing $H$ and makes a demand $V_{M}-\delta$ in $L H$. When agents $B$ update, they will choose $H$ since all agents $A$ have accepted $V_{M}-\delta$. When agents $A$ revise they will play $H$ since $V_{H}-x_{H H}>c+\delta$. Hence, the process reaches a new equilibrium $\theta^{\prime} \in \Sigma_{H}$ where $\rho\left(\theta^{\prime}\right)=\left\{H H,\left(V_{H}-x_{H H}\right), x_{H H}\right\}$.

(b) Consider $\Gamma_{U G}$ and let $\theta$ be an equilibrium belonging to $\Sigma_{H L}$. At $\theta$ the pair of demands $\left(y_{H L}, V_{M}-y_{H L}\right)$ is observed. Suppose that $y_{H L}<V_{M}-\delta$. By drifting, all agents $B$ are led to accept the maximum feasible demand made by $A$ in $H L$ and deem that all $A$ make demand larger than $V_{H}-c+\delta$ at $H H$. A new equilibrium $\theta_{1} \in \Sigma(\theta)$ is thus reached. Suppose now a single agent $A$ changes her demand from $y_{H L}$ to $V_{M}-\delta$. When agents $A$ update, they observe that all $B s$ have accepted the demand $V_{M}-\delta$; therefore in $H L$ their best response is $y_{H L}=V_{M}-\delta$. When agents $B$ update they continue to play $L$ since $\widetilde{x}_{H H}^{i}\left(\theta_{1}\right)<c+\delta$ holds for all $B s$. Hence, the process reaches a new equilibrium $\theta^{\prime} \in \Sigma_{H L}$ where $\rho\left(\theta^{\prime}\right)=\left\{H L,\left(V_{M}-\delta\right), \delta\right\}$. From $\theta^{\prime}$, by a sequence of single mutations, the process can reach an equilibrium $\theta^{*} \in \Sigma\left(\theta^{\prime}\right)$ in which all agents $A$ have beliefs: (i) $\widetilde{y}_{H H}^{i}\left(\theta^{*}\right)=y_{H H}$; (ii) $\widetilde{y}_{L H}^{i}\left(\theta^{*}\right)=\delta$; (iii) $\delta+c<y_{H H}<V_{H}-c-\delta$. Suppose now, an agent $B$ mutates by playing $H$ and making a demand $V_{H}-y_{H H}$ at $H H$. Let all agents $B$ revise; they will choose $H$ and ask $V_{H}-y_{H H}$. When agents $A$ update, the process reaches a new equilibrium $\theta^{\prime} \in \Sigma_{H}$ in which the pair of demands is $\left(y_{H H}, V_{M}-y_{H H}\right)$. Of course only the last sequence of mutations is required when $y_{H L}=V_{M}-\delta$.

The case in which $\theta$ is an equilibrium belonging to $\Sigma_{L H}$ is similar. Moreover the same argument holds true also for $\Gamma_{D G}$ with the caveat that any equilibrium belonging to $\Sigma_{H L}$ now supports the outcome $\left(V_{M}-\delta, \delta\right)$ only.

Proof of Lemma 3. Let $\theta \in \Sigma_{L}$. From $\theta$, by a sequence of single mutations, the process can reach a new equilibrium $\theta^{*} \in \Sigma(\theta)$ in which: at the subgame $\left(L H, V_{M}-y_{L H}\right)$ each agent $A$ accepts (i.e. she chooses $y_{L H}$ ); for ever $A, \widetilde{y}_{H H}^{i}\left(\theta^{*}\right)-y_{L H}-c<0$ but $V_{M}-y_{L H}-c>0$ for ever $B$. Suppose an agent $B$ mutates by playing $H$ and makes a 
demand $V_{M}-y_{L H}$ in $L H$. When all agents $B$ update, they will choose $H$ since population $A$ has accepted demand $V_{M}-y_{L H}$. When agents $A$ revise they will continue to play $L$ since $\widetilde{y}_{H H}^{i}\left(\theta^{*}\right)-y_{L H}-c<0$. Hence the process reaches a new equilibrium $\theta^{\prime} \in \Sigma_{L H}$ in which the pair of demands is $\left(y_{L H}, V_{M}-y_{L H}\right)$. The case in which $\theta$ is an equilibrium belonging to $\Sigma_{H L}$ is similar.

Lemma 1 and Lemma 2 together assert that, in both games, the adaptive process can lead to an equilibrium $\theta \in \Sigma_{H}$ by a sequence of single mutations, starting from any limit set $\Omega \notin \Sigma_{H}$. Therefore, according to Proposition 1 of Noldeke and Samuelson (1993), if $\Sigma_{S}$ is a strictly subset of $\Sigma$ then $\Sigma_{S} \subseteq \Sigma_{H}$.

Both Proposition 3 and Proposition 4 in the main text stem from a direct application of Theorem 2 of Ellison (2000) which we now briefly recall. Let $\Sigma$ be a union of limit sets; these sets can be either mutation connected or not. The Radius $R(\Sigma)$ is the minimum number of mutations needed to escape from the basin of attraction of $\Sigma$ and enter into another one with positive probability. Consider an arbitrary state $\theta \notin \Sigma$ and let $\left(m_{1}, m_{2}, . ., m_{T}\right)$ be a path from $\theta$ to $\Sigma$ where $\Omega_{1}, \Omega_{2}, \ldots \Omega_{r}$ is the sequence of limit sets through which the path passes consecutively. Obviously $\Omega_{i} \notin \Sigma$ for $i<r$ and $\Omega_{r} \subset \Sigma$. Furthermore, notice that a limit set can appear several times in this sequence but not consecutively. The modified cost of this path is defined by:

$$
c^{*}\left(m_{1}, \ldots, m_{T}\right)=c\left(m_{1}, . ., m_{T}\right)-\sum_{i=2}^{r-1} R\left(\Omega_{i}\right)
$$

where $c\left(m_{1}, . ., m_{T}\right)$ is the total number of mutations over the path $\left(\theta, m_{1}, m_{2}, . ., m_{T}\right)$. Let $c^{*}(\theta, \Sigma)$ be the minimal modified cost among all paths from $\theta$ to $\Sigma$. The Modified Coradius of the basin of attraction of $\Sigma$ is then:

$$
C R^{*}(\Sigma)=\max _{\theta \notin \Sigma} c^{*}(\theta, \Sigma)
$$

Theorem 2 of Ellison (2000) shows that every union of limit sets $\Sigma$ with $R(\Sigma)>C R^{*}(\Sigma)$ encompasses all the stochastically stable states. In order to compute the minimum number of mutations needed to escape from an equilibrium belonging to $\Sigma_{H}$, both Propositions 3 and 4 make use of the result stated in Lemma 7 below. In what follows we write $\theta_{x}$ as shorthand for an equilibrium belonging to $\Sigma_{H}$ with $\left(V_{H}-x, x\right)$ as the distributional rule.

Lemma 7 For $\delta$ sufficiently small, the minimum number of mutations needed to get from $\Sigma\left(\theta_{x}\right)$ to an equilibrium with the same investment profile but different demands is:

$$
\begin{array}{lll}
r_{B}^{+}(x)=\left\lfloor N\left(\frac{\delta}{V_{H}-x}\right)\right] & \text { if } & x<\frac{V_{H}}{2} \\
r_{A}^{-}(x)=\left\lfloor N\left(\frac{\delta}{x}\right)\right] & \text { if } & x>\frac{V_{H}}{2}
\end{array}
$$

where $r_{B}^{+}(x)$ is the number of mutations needed for the transition from $\theta_{x}$ to $\theta_{x+\delta}$ whereas $r_{A}^{-}(x)$ is the number of mutations needed for the transition from $\theta_{x}$ to $\theta_{x-\delta}$. Moreover, $r_{B}^{+}(x)$ is a strictly increasing function of $x$ and $r_{A}^{-}(x)$ is a strictly decreasing function of $x$.

Proof: By a direct application of Young (1993)

Before giving the proof of Proposition 3 two further preliminary results are needed. These are provided by Lemma 8 and Lemma 9 below. The first allows us to argue that a norm of cooperation supporting the efficient investment profile evolves in the long run when condition (5) holds. Given this, the second result allows us to detect which norm of distribution arises when the stochastically stable outcome support the efficient investment profile. 
Lemma 8 Consider $\Gamma_{U G}$. Then:

(a) an equilibrium $\theta^{\prime} \in \Sigma_{L}$ can be reached from $\theta \in \Sigma_{C H}^{U}$ by a sequence of singlemutation transitions:

(b) under condition (5), $C R^{*}\left(\Sigma_{I H}^{U}\right)=1$;

(c) under condition (5), $R\left(\Sigma_{I H}^{U}\right)>1$ and, consequently, $\Sigma_{S} \subseteq \Sigma_{I H}^{U}$.

Proof. Point (a). Consider some $\theta \in \Sigma_{C H}^{U}$ and let $\left\{V_{H}-x, x\right\}$ be the observed pair of demands. We show that starting from $\Sigma_{C H}^{U}$ it may be possible to enter into the basin of attraction of an equilibrium $\theta^{\prime} \in \Sigma_{L}$ through a sequence of single-mutation transitions. In order to describe this transition four cases have to be taken into account: $(1) x>x_{A}^{U} ;(2)$ $x=x_{A}^{U}$; (3) $x<x_{B}^{U}$; (4) $x=x_{B}^{U}$. We give the proof for cases (1) and (2) only; the remaining cases are symmetric.

Case (1): let $x>x_{A}^{U}$. At $\theta$ the following inequality must hold:

$$
\begin{array}{ll}
\text { Population } A: & \text { Population } B: \\
\left(V_{H}-x-c\right)-\widetilde{y}_{L H}^{i}(\theta) \geq 0 & x-c-\widetilde{x}_{H L}^{i}(\theta) \geq 0 \\
V_{H}-x-c<V_{M}-\delta & x-c>V_{M}-\delta .
\end{array}
$$

From $\theta$ the process can reach a new equilibrium $\theta_{1} \in \Sigma(\theta)$ by a sequence of single mutations in which the following is true for every agent: (i) $\widetilde{x}_{L H}^{i}\left(\theta_{1}\right)=\delta$ and (ii) $\widetilde{y}_{H L}^{i}\left(\theta_{1}\right)-c<0$. Suppose an agent $A$ mutates by playing $L$ and accepting her opponent's demand at $L H$. Let all agents $A$ update. Since the mutant receives $V_{M}-\delta$, all $A s$ imitate and play $L$. When agents $B$ revise they will play $L$. The process then reaches a new equilibrium $\theta^{\prime} \in \Sigma_{L}$.

Case (2): let $x=x_{A}^{U}$. At $\theta$, for any agent $A$ it must be true that $V_{H}-x_{A}^{U}-c=V_{M}-\delta$. From $\theta$ the process can reach a new equilibrium $\theta_{1} \in \Sigma(\theta)$ by a sequence of single mutations in which the following is true for every agent: (i) $\widetilde{x}_{L H}^{i}\left(\theta_{1}\right)=x_{L H}$; (ii) $x_{L H}-c<0$; (iii) $\widetilde{y}_{L H}^{i}\left(\theta_{1}\right)=V_{M}-\delta$ and (iv) $\widetilde{y}_{H L}^{i}\left(\theta_{1}\right)-c<0$. Suppose an agent $B$ mutates by demanding $x^{\prime}>x_{A}^{U}$ at $H H$. When agents $A$ update they will all choose $L$ since, for whatever best action at $H H$, the expected payoff by playing $H$ is now smaller than $V_{M}-\delta$. When all agents $B$ revise they will play $L$. The process then reaches a new equilibrium $\theta^{\prime} \in \Sigma_{L}$.

Point (b). Under condition (5) $\Sigma_{I H}^{U}$ is well defined. By a direct application of previous point (a), along with point (a) of Lemma 2, it follows that from $\theta \in \Sigma_{C H}^{U}$ it is possible to reach $\widehat{\theta} \in \Sigma_{I H}^{U}$ through a sequence of single mutations. Therefore $c^{*}\left(\theta, \Sigma_{I H}^{U}\right)=1$ for any $\theta \in \Sigma_{C H}^{U}$

Besides, from Lemmas 1 and 2 , we can deduce that for any $\Omega \notin \Sigma_{H}$ the minimal modified cost for all paths from $\Omega$ to $\Sigma_{I H}$, is equal to one, whatever the number of limit sets the path goes through may be. Therefore, by putting together these results, we get:

$$
C R^{*}\left(\Sigma_{I H}^{U}\right)=\max _{\Omega \notin \Sigma_{I H}^{U}} c^{*}\left(\Omega, \Sigma_{I H}^{U}\right)=1 .
$$

Point (c). We show that, under condition (5), more than one mutation is needed to leave the basin of attraction of $\Sigma_{I H}^{U}$ even when the worst-case equilibrium scenario is considered as starting state.

I) First we show that a single mutation from $H$ to $L$ does not enable the process to leave the set $\Sigma_{I H}^{U}$ even if at $\theta_{x} \in \Sigma_{I H}^{U}$ each agent expects to receive: (i) the maximum payoff when she plays $L$ but the opponent still plays $H$; (ii) the minimum payoff when she plays $H$ but the opponent shifts to $L$.

Let us consider this scenario and suppose that an agent $B$ had switched from $H$ to $L$ and all agents $A$ revised. This updating does not cause agents $A$ to play $L$ if

$$
\frac{N-1}{N}\left[\left(V_{H}-x-c\right)-\left(V_{M}-\delta\right)\right]+\frac{1}{N}(\delta-c)>0
$$

which can be rewritten as

$$
\frac{N-1}{N}\left(V_{H}-x\right)-c>\frac{N-1}{N} V_{M}-\delta .
$$


Since $x \in\left[\widehat{x}_{B}^{U}, \widehat{x}_{A}^{U}\right]$, condition (13) holds by definition. Therefore no agent $A$ will change her action after the revision. Similar argument can be applied to population $B$. Since as soon as the mutant revises the process returns to $\Sigma_{I H}^{U}$, then a single mutation from $H$ to $L$ is not enough to leave the basin of attraction of $\Sigma_{I H}^{U}$.

II) We now show that a single mutation from $x$ to $x^{\prime}$ (resp. from $V_{H}-x$ to $y^{\prime}$ ) does not enable the process to run away from the basin of attraction of $\Sigma_{I H}^{U}$ even if at $\theta_{x} \in \Sigma_{I H}^{U}$ each agent expects to get the maximum payoff when she plays $L$ and the opponent chooses $H$. Let one agent $B$ only change her demand to $x^{\prime}$. Obviously, no agents $B$ imitate the mutant when revising. When $A s$ update we know from Lemma 4 that their best response is either $V_{H}-x$ or $V_{H}-x^{\prime}$. If $x^{\prime}>x$, agent $A$ expects to receive $\left(V_{H}-x\right) \frac{N-1}{N}-c$ when she demands $V_{H}-x$ and $V_{H}-x^{\prime}-c$ when she demands $V_{H}-x^{\prime}$. Under Assumption 2, the former payoff is greater than the latter. Hence agents $A$ will not change their demands when updating. Moreover, since $\left(V_{H}-x\right) \frac{N-1}{N}-c \geq V_{M}-\delta$, then updating will not cause agents $A$ to play action $L$. If $x^{\prime}<x$, agent $A$ expects to get $V_{H}-x-c$ when she demands $V_{H}-x$ and $\frac{1}{N}\left(V_{H}-x^{\prime}\right)-c$ when she demands $V_{H}-x^{\prime}$. Under Assumption 2, the former payoff is greater than the latter. Hence, agents $A$ will not change their demands when updating. Moreover, since $V_{H}-x-c>V_{M}-\delta$, then updating will not cause agents $A$ to play action $L$. The case in which an agent $A$ mutates from $V_{H}-x$ to $y^{\prime}$ is symmetric. Since as soon as the mutant revises the process returns to $\Sigma_{I H}^{U}$, then a single mutation from $x$ to $x^{\prime}$ (resp. from $V_{H}-x$ to $y^{\prime}$ ) is not enough to escape from the basin of attraction of $\Sigma_{I H}^{U}$.

Points I) and II) taken together say that more than one mutation is needed in order to escape from the basin of attraction of $\Sigma_{I H}^{U}$, i.e. $R\left(\Sigma_{I H}^{U}\right)>1$. Given that $C R^{*}\left(\Sigma_{I H}^{U}\right)=1$, by Theorem 2 of Ellison (2000) we get $\Sigma_{S} \subseteq \Sigma_{I H}^{U}$.

We have now to derive the norms of distribution supporting $\Sigma_{I H}^{U}$. In order to do this we have: (i) to compute the radius of $\Sigma\left(\theta_{x}\right)$, i.e. the minimum number of mutations required to destabilize the outcome supported by $\theta_{x}, \forall \theta_{x} \in \Sigma_{I H}^{U}$; (ii) to find an equilibrium belonging to $\Sigma_{I H}^{U}$ such that $R\left(\Sigma\left(\theta_{x}\right)\right)>C R^{*}\left(\Sigma\left(\theta_{x}\right)\right)$. Lemma 7 provides the minimum number of mutations required to make a transition from $\theta_{x} \in \Sigma_{I H}^{U}$ to another equilibrium supporting the same investment profile $H H$, but a different distributional rule. Lemma 9 below completes all the required details by giving the minimum number of mutations required to make a transition from $\theta_{x} \in \Sigma_{I H}^{U}$ to $\theta^{\prime} \notin \Sigma_{I H}^{U}$.

Lemma 9 Consider $\Gamma_{U G}$. The minimum number of mutations required to get from $\theta_{x} \in$ $\Sigma_{I H}^{U}$ to an equilibrium which supports a different investment profile is:

$$
\begin{aligned}
& \bar{r}_{A}(x)=\left\lfloor N\left(1-\frac{V_{M}-\delta+c}{x}\right)\right\rfloor \quad \text { if } \quad x<\frac{V_{H}}{2} \\
& \bar{r}_{B}(x)=\left\lfloor N\left(1-\frac{V_{M}-\delta+c}{V_{H}-x}\right)\right\rceil \text { if } \quad x>\frac{V_{H}}{2}
\end{aligned}
$$

Proof. Consider $\theta_{x} \in \Sigma_{I H}^{U}$. Suppose $p_{1}$ agents $B$ mutate by playing $L$ and $p_{2}$ agents $B$ mutate by claiming $x^{\prime}>x_{A}^{U}$. For a given pair $\left(p_{1}, p_{2}\right)$, agents $A$ have the largest incentive to change into $L$ if their beliefs are such that: (i) they expect to get the maximum payoff in an $L H$ match; (ii) they expect to obtain the minimum payoff in an $H L$ match. Consider equilibrium $\theta_{x} \in \Sigma_{I H}^{U}$ in which the following holds for all agents: (i); $\widetilde{y}_{L H}^{i}=V_{M}-\delta$ and $\widetilde{y}_{H L}^{i}=\delta$; (ii) $\widetilde{x}_{L H}^{i}=\delta$ and in the subgame $\{H L, \delta\}$ all agents $B$ accept. At $\theta_{x}$, when some agents $B$ mutate and these mutations induce all agents $A$ to play $L$, the process enters into the basin of attraction of equilibrium $\theta^{\prime} \in \Sigma_{L}$ with positive probability. Sure enough, after updating, all agents $A$ decide to play $L$ if

$$
\frac{N-p_{1}}{N}\left(V_{M}-\delta\right)>\mu_{H}\left(\widetilde{\theta}_{x}, p_{1}, p_{2}\right)
$$


where LHS is the expected payoff by playing $L$ and RHS is the expected payoff by playing $H$. However, $\mu_{H}\left(\widetilde{\theta}_{x}, p_{1}, p_{2}\right)$ depends on what the best demand in a match $H H$ is. In particular

$$
\mu_{H}(.)=\left\{\begin{array}{c}
\frac{N-p_{2}-p_{1}}{N}\left(V_{H}-x\right)+\frac{p_{1}}{N} \delta-c \text { if } \frac{N-p_{2}-p_{1}}{N-p_{1}}\left(V_{H}-x\right) \geq\left(V_{H}-x^{\prime}\right) \\
\frac{N-p_{1}}{N}\left(V_{H}-x^{\prime}\right)+\frac{p_{1}}{N} \delta-c \text { if } \frac{N-p_{2}-p_{1}}{N-p_{1}}\left(V_{H}-x\right)<\left(V_{H}-x^{\prime}\right)
\end{array}\right.
$$

The minimum number of mutations in population $B$ comes from the comparison between the solutions of two constraint minimization problems ( $M 1$ and $M 2)$. In both problems the objective function is $p_{1}+p_{2}$. In the first (resp. second) problem we contemplate the case in which the best action in $H H$ is $V_{H}-x^{\prime}$ (resp. $V_{H}-x$ ). Both problems require $p_{1}=0$ as a solution. Moreover $p_{2}^{M 1}=N\left(\frac{x^{\prime}-x}{V_{H}-x}\right)$ is the solution to the first problem and $p_{2}^{M 2}=N\left(1-\frac{V_{M}-\delta+c}{V_{H}-x}\right)$ is the solution to the second. Since $p_{2}^{M 1}>p_{2}^{M 2}$, the minimum number of mutations in population $B$ involves that: (i) mutating agents only change their demands in the $H H$ profile; (ii) these mutations cause agent $A$ to shift to action $L$ when the best action in $H H$ continues to be $V_{H}-x$. Hence:

$$
\bar{r}_{B}(x)=\left\lfloor N\left(1-\frac{V_{M}-\delta+c}{V_{H}-x}\right)\right\rceil
$$

and

$$
\bar{r}_{B}=\min _{x} \bar{r}_{B}(x)=\bar{r}_{B}\left(\widehat{x}_{A}\right) .
$$

Suppose now some agents $A$ mutate. As before, two kinds of mutations must be considered: $p_{1}$ agents $A$ mutate by playing $L$ and $p_{2}$ agents $A$ mutate by demanding $V_{H}-x^{\prime}$ where $x^{\prime}<x_{B}^{U}$. In this case we look for an equilibrium $\theta_{x} \in \Sigma_{I H}^{U}$ in which for all agents: (i) $\widetilde{x}_{L H}^{i}=\delta$ and $\widetilde{x}_{H L}^{i}=V_{M}-\delta$; (ii) $\widetilde{y}_{H L}^{i}=\delta$ and in the subgame $\{L H, \delta\}$ all agents $A$ accept. It is easy to see that if some mutations of agents $A$ occurs at $\theta_{x}$ and these mutations induce all agents $B$ to play $L$, then with positive probability the process enters into the basin of attraction of equilibrium $\theta^{\prime} \in \Sigma_{L}$. After updating all agents $B$ decide to play $L$ if

$$
\frac{N-p_{1}}{N}\left(V_{M}-\delta\right)>\mu_{H}\left(\widehat{\theta}_{x}, p_{1}, p_{2}\right)
$$

where

$$
\mu_{H}(.)=\left\{\begin{array}{l}
\frac{N-p_{2}-p_{1}}{N} x+\frac{p_{1}}{N} \delta-c \text { if } \frac{N-p_{2}-p_{1}}{N-p_{1}} x \geq x^{\prime} \\
\frac{N-p_{1}}{N} x^{\prime}+\frac{p_{1}}{N} \delta-c \text { if } \frac{N-p_{2}-p_{1}}{N-p_{1}} x<x^{\prime} .
\end{array}\right.
$$

Proceeding as before, the minimum number of mutations in population $A$ is

$$
\bar{r}_{A}(x)=\left\lfloor N\left(1-\frac{V_{M}-\delta+c}{x}\right)\right\rceil
$$

and

$$
\bar{r}_{A}=\min _{x} \bar{r}_{A}(x)=\bar{r}_{A}\left(\widehat{x}_{B}\right)
$$

By comparing (14) and (15) we obtain $\bar{r}_{B}(x)<\bar{r}_{A}(x)$ if $x>\frac{V_{H}}{2}$

Proof of Proposition 3. Point (a). Consider $\Gamma_{U G}$. From Point (c) of Lemma 8 we know that $\Sigma_{S} \subseteq \Sigma_{I H}^{U}$ when condition 5 holds. Therefore, we are only left with the task of deriving the distributional norm supporting the equilibria belonging to $\Sigma_{I H}^{U}$.

To detect $R\left(\Sigma\left(\theta_{x}\right)\right)$ for any $\theta_{x} \in \Sigma_{I H}^{U}$ we compare the results coming from Lemma 7 with those coming from Lemma 9 . Notice that $r_{B}^{+}(x) \leq \bar{r}_{B}(x)$ if $V_{M}-\delta \leq V_{H}-(x+\delta)-c$ 
and $r_{A}^{-}(x) \leq \bar{r}_{A}(x)$ if $V_{M}-\delta \leq x-\delta-c$; since these conditions are always satisfied for any $x \in\left[\widehat{x}_{B}^{U}, \widehat{x}_{A}^{U}\right]$ we conclude that

$$
R\left(\Sigma\left(\theta_{x}\right)\right)=\left\{\begin{array}{lll}
r_{B}^{+}(x) & \text { if } & x<\frac{V_{H}}{2} \\
r_{A}^{-}(x) & \text { if } & x>\frac{V_{H}}{2} .
\end{array}\right.
$$

Let $\bar{x} \equiv \frac{V_{H}}{2}$ and consider the set of equilibria $\Sigma\left(\theta_{\bar{x}}\right)$. Let $\theta_{x} \in \Sigma_{I H}^{U}$ be an equilibrium. When $x<\bar{x}$, then the minimal modified cost from $\theta_{x}$ to $\Sigma\left(\theta_{\bar{x}}\right)$ is associated with the path $\theta_{x} \rightarrow \theta_{x+\delta} \rightarrow \ldots \rightarrow \theta_{\bar{x}-\delta} \rightarrow \Sigma\left(\theta_{\bar{x}}\right)$. Conversely, when $x>\bar{x}$, the minimal modified costs is associated with path $\theta_{x} \rightarrow \theta_{x-\delta} \rightarrow \ldots \rightarrow \theta_{\bar{x}+\delta} \rightarrow \Sigma\left(\theta_{\bar{x}}\right)$. Hence

$$
\begin{aligned}
& c^{*}\left(\theta_{x} ; \Sigma\left(\theta_{\bar{x}}\right)\right)=r_{B}^{+}(x) \quad \text { if } \quad x<\frac{V_{H}}{2} \\
& c^{*}\left(\theta_{x} ; \Sigma\left(\theta_{\bar{x}}\right)\right)=r_{A}^{-}(x) \quad \text { if } \quad x>\frac{V_{H}}{2} .
\end{aligned}
$$

By the monotonicity of $r_{B}^{+}(x)$ and $r_{A}^{-}(x)$ we obtain

$$
C R^{*}\left(\Sigma\left(\theta_{\bar{x}}\right)\right)=\max \left(r_{B}^{+}(\bar{x}-\delta) ; r_{A}^{-}(\bar{x}+\delta)\right) .
$$

Since

$$
R\left(\Sigma\left(\theta_{\bar{x}}\right)\right)=r_{B}^{+}(\bar{x})=r_{A}^{-}(\bar{x})>C R^{*}\left(\Sigma\left(\theta_{\bar{x}}\right)\right)
$$

it follows from Ellison (2000) that the only stochastically stable outcome is $\left\{H H, \frac{V_{H}}{2}, \frac{V_{H}}{2}\right\}$.

Point (b). Recall that when condition (5) does not hold then $\Sigma_{H}=\Sigma_{C H}^{U}$; hence one mutation is enough to exit from the basin of attraction of $\Sigma_{H}$ (Point (a) of Lemma 8). In what follow we shall apply Theorem 3 of Ellison (2000). It is worth noticing that $R(\Omega)=1$ for any limit set $\Omega$. Indeed let $\Omega^{\prime}$ be a limit set; then it is always possible to reach $\Omega^{*} \in \Sigma(\Omega)$ with one mutation by letting one agent to drift at some unreached information set. Hence, if for limit sets $\Omega$ and $\Omega^{\prime}$ we have $C R^{*}\left(\Omega^{\prime}\right)=1$, then $\mu_{*}(\Omega)>0$ implies that $\mu_{*}\left(\Omega^{\prime}\right)>0$. Concerning the minimal modified cost among all paths from a generic limit $\Omega$ set we already know that:

(i) if $\rho(\Omega)$ is not a singleton, at least one $\Omega^{\prime}$ exists with $\rho\left(\Omega^{\prime}\right)$ singleton, such that $C R^{*}\left(\Omega^{\prime}\right)=1$ (Lemma 1$)$;

(ii) if $\Omega \in \Sigma_{L}$, at least two limit sets $\Omega^{\prime}$ and $\Omega^{*}$ exist, with different distributional rules but both belonging to either $\Sigma_{H}$ or $\left(\Sigma_{H L} \cup \Sigma_{L H}\right)$ and such that $C R^{*}\left(\Omega^{\prime}\right)=C R^{*}\left(\Omega^{*}\right)=1$ (Point (a) of Lemma 2 and Lemma 3);

(iii) if $\Omega \in \Sigma_{H L}\left(\operatorname{resp} . \Sigma_{L H}\right)$, at least two limit set $\Omega^{\prime}$ and $\Omega^{*}$ exist, with different distributional rules but both belonging to $\Sigma_{H}$ and such that $C R^{*}\left(\Omega^{\prime}\right)=C R^{*}\left(\Omega^{*}\right)=1$ (Point (b) of Lemma 2);

(iv) if $\Omega \in \Sigma_{H}$, at least one limit set $\Omega^{\prime} \in \Sigma_{L}$ exists such that $C R^{*}\left(\Omega^{\prime}\right)=1$ (Point (a) of Lemma 8).

Let $\Omega$ be a limit set such that $\mu_{*}(\Omega)>0$. By collecting previous information and using Theorem 3 of Ellison (2000) we conclude that: (i) if $\rho(\Omega)$ is not a singleton, then $\mu_{*}\left(\Omega^{\prime}\right)>0$ where $\rho\left(\Omega^{\prime}\right)$ is a singleton; (ii) if $\Omega \in \Sigma_{L}$, then $\mu_{*}\left(\Omega^{\prime}\right)>0$ and $\mu_{*}\left(\Omega^{*}\right)>0$ where $\Omega^{\prime}$ and $\Omega^{*}$ both belong to either $\Sigma_{H}$ or $\Sigma_{H L} \cup \Sigma_{L H}$; (iii) if $\Omega \in \Sigma_{H L}$ (resp. $\left.\Sigma_{L H}\right)$ then $\mu_{*}\left(\Omega^{\prime}\right)>0$ and $\mu_{*}\left(\Omega^{*}\right)>0$ where both $\Omega^{\prime}$ and $\Omega^{*}$ belong to $\Sigma_{H}$; (iv) if $\Omega \in \Sigma_{H}$ then $\mu_{*}\left(\Omega^{\prime}\right)>0$ where $\Omega^{\prime} \in \Sigma_{L}$. Hence in this case an investment norm and a bargaining norm cannot evolve in the long run.

We now turn our attention to game $\Gamma_{D G}$ and to the proof of Proposition 4. As for game $\Gamma_{U G}$, also in this case two preliminary results are needed. In Lemma 10 below we show that $\Sigma_{S} \subseteq \Sigma_{I H}^{D}$

Lemma 10 Consider $\Gamma_{D G}$. Then:

(a) $C R^{*}\left(\Sigma_{I H}^{D}\right)=1$;

(b) $R\left(\Sigma_{I H}^{D}\right)>1$ and, consequently, $\Sigma_{S} \subseteq \Sigma_{I H}^{D}$. 
Proof. Point (a). Firstly notice that the set $\Sigma_{I H}^{D}$ is always well-defined since we can always find a sequence of $\left\{\delta_{i}\right\}$ converging to zero compatible with condition (10). Indeed it is enough to consider $\delta_{i}<\delta_{I H}$ where $0<\delta_{I H} \leq \frac{V_{H}-2 c}{4}$. Secondly observe that $\Sigma_{C H}^{D}$ only supports two distributional rules, namely, $x_{B}^{D}$ and $x_{A}^{D}$. Consider an equilibrium $\theta \in \Sigma_{C H}^{D}$ with $x_{B}^{D}$ as distributional rule and suppose a single mutation from $V_{H}-x_{B}^{D}$ to $V_{H}-x^{\prime}>$ $V_{H}-x_{B}^{D}$ occurs in population $A$. Suppose all $B s$ revise. Notice that whatever the best reply at $H H$ is, the expected payoff by playing $H$ is now smaller than $\delta$. Hence updating will now cause agents $B$ to change investment action and to play $L$. Therefore when $\theta_{x} \in \Sigma_{C H}^{D}$ one mutation is enough to enter into the basin of attraction of $\theta^{\prime} \in\left(\Sigma_{H L} \cup \Sigma_{L H}\right)$. A similar conclusion holds also when we consider $x_{A}^{D}$ as distributional rule. Hence, from Lemma 2 point (b) we can deduce that the minimal modified cost across all paths from $\theta_{x} \in \Sigma_{C H}^{D}$ to $\Sigma_{I H}^{D}$ is equal to one, whatever the number of limit sets the path goes through.

In addition, from Lemma 1 and from Point (b) of Lemma 2, we can deduce that, for any $\Omega \notin \Sigma_{H}$, the minimal modified cost for all paths from $\theta$ to $\Sigma_{I H}^{D}$ is equal to one, whatever the number of limit sets the path goes through may be. Therefore

$$
C R^{*}\left(\Sigma_{I H}^{D}\right)=\max _{\Omega \notin \Sigma_{I H}^{U}} c^{*}\left(\Omega, \Sigma_{I H}^{D}\right)=1 .
$$

Point (b). Let $\theta_{x} \in \Sigma_{I H}^{D}$ and consider the worst-case equilibrium scenario in which $\widetilde{y}_{L H}^{i}\left(\theta_{x}\right)=V_{H}-x-c$ and $\widetilde{x}_{H L}^{i}\left(\theta_{x}\right)=x-c$. Suppose a single mutation from $H$ to $L$ occurs in population $B$. This implies that profile $H L$ is reached in which agents $A$ behave as dictators and claim $V_{M}-\delta$. When agents $B$ revise, their updated beliefs become $\widetilde{x}_{H L}^{i}=\delta$; as a consequence they choose $H$. The process then returns to an equilibrium $\theta^{\prime} \in \Sigma(\theta)$. The same occurs also when a single mutation from $H$ to $L$ occurs in population $A$. Suppose now a single agent $B$ mutates her demand from $x$ to $x^{\prime}$. Obviously no agent $B$ imitates the mutant when updating. When agents $A$ revise, we know from Lemma 4 that their best response is either $V_{H}-x$ or $V_{H}-x^{\prime}$. When $x^{\prime}>x$, agent $A$ expects to receive $\left(V_{H}-x\right) \frac{N-1}{N}-c$ by claiming $V_{H}-x$ and expect to receive $V_{H}-x^{\prime}-c$ by asking $V_{H}-x^{\prime}$. When instead $x^{\prime}<x$, agent $A$ expects to receive $V_{H}-x-c$ by claiming $V_{H}-x$ and expect to receive $\left(V_{H}-x^{\prime}\right) \frac{1}{N}-c$ by asking $V_{H}-x^{\prime}$. It is simple to see that, whatever the relation between $x$ and $x^{\prime}$ is, Assumption 2 implies that the best response is always $V_{H}-x$. Hence, updating will not cause agents $A$ to change both claim and investment action. This result allow us to assert that $R\left(\Sigma_{I H}^{D}\right)>1$. Therefore, given that $C R^{*}\left(\Sigma_{I H}^{D}\right)=1$, by using Theorem 2 of Ellison (2000) we get $\Sigma_{S} \subseteq \Sigma_{I H}^{D}$.

We have now to derive the norms of distribution supporting $\Sigma_{I H}^{D}$. As for $\Gamma_{U G}$, we have to compute the radius of $\theta_{x} \in \Sigma_{I H}^{D}$ (i.e. the minimum number of mutations required to destabilize the outcome supported by $\left.\theta_{x}, \forall \theta_{x} \in \Sigma_{I H}^{D}\right)$, and to find an equilibrium belonging to $\Sigma_{I H}^{D}$ such that $R\left(\Sigma\left(\theta_{x}\right)\right)>C R^{*} \Sigma\left(\theta_{x}\right)$. The relevant information is provided by Lemma 7 , which continues to be true, and by Lemma 11 below.

Lemma 11 Consider $\Gamma_{D G}$. The minimum number of mutations required to get from $\theta_{x} \in$ $\Sigma_{I H}^{D}$ to an equilibrium which supports a different investment profile is:

$$
\begin{aligned}
& \bar{r}_{A}(x)=\left\lfloor N\left(1-\frac{\delta+c}{x}\right)\right\rceil \\
& \bar{r}_{B}(x)=\left\lfloor N\left(1-\frac{\delta+c}{V_{H}-x}\right)\right\rceil .
\end{aligned}
$$

Proof. We give proof for $\bar{r}_{B}(x)$ only; similar arguments can be used for $\bar{r}_{A}(x)$. Consider $\theta_{x} \in \Sigma_{I H}^{D}$. Suppose $p_{1}$ agents $B$ mutate by playing $L$ and $p_{2}$ agents $B$ mutate by claiming $x^{\prime}>\widehat{x}_{A}^{D}$ let $\widetilde{\theta}_{x}$ be the resulting state. Suppose these mutations induce all agents $A$ to play $L$. Suppose all $A$ believe to receive at $L H$ a payoff $\widetilde{y}_{L H}$; this belief is compatible with the fact that $\theta_{x}$ is an equilibrium only if $\widetilde{y}_{L H} \leq V_{H}-x-c$. Sure enough, at $\widetilde{\theta}_{x}$ all agents $A$ decide to play $L$ if, after updating,

$$
\frac{N-p_{1}}{N} \widetilde{y}_{L H}>\mu_{H}\left(\widetilde{\theta}_{x}, p_{1}, p_{2}\right)
$$


where the LHS is the expected payoff by playing $L$ and the RHS is the expected payoff by playing $H$. However, $\mu_{H}\left(\widetilde{\theta}_{x}, p_{1}, p_{2}\right)$ depends on the best demand in an $H H$ match. In particular

$$
\mu_{H}(.)=\left\{\begin{array}{l}
\frac{\left(N-p_{2}-p_{1}\right)\left(V_{H}-x\right)}{N}+\frac{p_{1}\left(V_{M}-\delta\right)}{N}-c \text { if } \frac{\left(N-p_{2}-p_{1}\right)\left(V_{H}-x\right)}{N-p_{1}} \geq\left(V_{H}-x^{\prime}\right) \\
\frac{\left(N-p_{1}\right)\left(V_{H}-x^{\prime}\right)}{N}+\frac{p_{1}\left(V_{M}-\delta\right)}{N}-c \text { if } \frac{\left(N-p_{2}-p_{1}\right)\left(V_{H}-x\right)}{N-p_{1}}<\left(V_{H}-x^{\prime}\right) .
\end{array}\right.
$$

Given $\widetilde{y}_{L H}$, the minimum number of mutations in population $B$ are obtained by solving two constrained minimization problems $(M 1$ and $M 2)$. In both problems, the objective function is $p_{1}+p_{2}$. In $M 1$ (resp. $M 2$ ), we contemplate the case in which the best action at $H H$ is $V_{H}-$ $x^{\prime}\left(\right.$ resp. $\left.V_{H}-x\right)$. Both problems require $p_{1}=0$ as a solution. Moreover, $p_{2}^{M 1}=N\left(\frac{x^{\prime}-x}{V_{H}-x}\right)$ is the solution of $M 1$ for whatever value of $\widetilde{y}_{L H}$, and $p_{2}^{M 2}\left(\widetilde{y}_{L H}\right)=N\left(1-\frac{\widetilde{y}_{L H}+c}{V_{H}-x}\right)$ is the solution of $M 2$. Notice that $p_{2}^{M 2}$ depends on $\widetilde{y}_{L H}$. Suppose $p_{2}^{M 2}\left(\widetilde{y}_{L H}\right)$ agents $B$ claim $x^{\prime}>x$. By updating, all agents $A$ play $L$ so that only profile $L H$ is observed. Since all agents $B$ claim $V_{M}-\delta$ then, after updating, all agents $A$ learn that $\widetilde{y}_{L H}=\delta$. This implies that no agent $A$ has the incentive to play $H$ if

$$
\frac{N-p_{2}^{M 2}\left(\widetilde{y}_{L H}\right)}{N}\left(V_{H}-x\right)-c \leq \delta,
$$

condition weakly satisfied when $\widetilde{y}_{L H}=\delta$. Therefore, in the second minimization problem the minimum number of mutations of agents $B$ needed to enter into the basin of attraction of $\theta^{\prime}$ from $\theta_{x}$ is

$$
p_{2}^{M 2}=N\left(1-\frac{\delta+c}{V_{H}-x}\right) .
$$

Since $p_{2}^{M 1}>p_{2}^{M 2}$, the minimum number of mutations involves that: (i) mutating agents only change their demands in the $H H$ profile; (ii) these mutations cause agent $A$ to shift to action $L$ when the best action in match $H H$ continues to be $V_{H}-x$; (iii) all agents $A$ correctly anticipate the distribution occurring at $L H$. Hence:

$$
\bar{r}_{B}(x)=\left\lfloor N\left(1-\frac{\delta+c}{V_{H}-x}\right)\right\rfloor .
$$

Proof of Proposition 4. Consider $\Gamma_{D G}$. From Lemma 10 we know that $\Sigma_{S} \subseteq \Sigma_{I H}^{D}$. Thus, we are only left with the task of deriving the distributional norm supporting the equilibria belonging to $\Sigma_{I H}^{D}$. To detect $R\left(\Sigma\left(\theta_{x}\right)\right)$ for any $\theta_{x} \in \Sigma_{I H}^{D}$ we compare the results coming from Lemma 7 with those coming from Lemma 11. Notice that $r_{B}^{+}(x) \leq \bar{r}_{B}(x)$ if $2 \delta \leq V_{H}-x-c$ and $r_{A}^{-}(x) \leq \bar{r}_{A}(x)$ if $2 \delta \leq x-c$; since these conditions are always satisfied for any $x \in\left[\widehat{x}_{B}^{D}, \widehat{x}_{A}^{D}\right]$ we conclude that

$$
R\left(\Sigma\left(\theta_{x}\right)\right)=\left\{\begin{array}{lll}
r_{B}^{+}(x) & \text { if } & x<\frac{V_{H}}{2} \\
r_{A}^{-}(x) & \text { if } & x>\frac{V_{H}}{2}
\end{array}\right.
$$

Before deriving $C R^{*}\left(\Sigma\left(\theta_{x}\right)\right)$, we observe that: $\widehat{x}_{B}^{D}<\frac{V_{H}}{2} \leq \widehat{x}_{A}^{D}$ if $\delta \leq \delta_{I H} \equiv \frac{V_{H}-2 c}{4}$. Therefore when $\Sigma_{I H}^{D}$ is well defined (see the argument at point (a) of Lemma 10) the condition $\widehat{x}_{B}^{D}<\frac{V_{H}}{2}<\widehat{x}_{A}^{D}$ always holds. Let $\bar{x} \equiv \frac{V_{H}}{2}$ and consider the set of equilibria $\Sigma\left(\theta_{\bar{x}}\right)$; let $\theta_{x} \in \Sigma_{I H}^{D}$ be an equilibrium with $x \neq \bar{x}$. From Lemma 7 we know that

$$
c^{*}\left(\theta_{x}, \Sigma\left(\theta_{\bar{x}}\right)\right)=\left\{\begin{array}{ccc}
r_{B}^{+}(x) & \text { if } & x<\frac{V_{H}}{2} \\
r_{A}^{-}(x) & \text { if } & x>\frac{V_{H}}{2} .
\end{array}\right.
$$


By the monotonicity of $r_{B}^{+}(x)$ and $r_{A}^{-}(x)$ we obtain

$$
C R^{*}\left(\Sigma\left(\theta_{\bar{x}}\right)\right)=\max \left(r_{B}^{+}(\bar{x}-\delta), r_{A}^{-}(\bar{x}+\delta)\right) .
$$

Of course, when $x=\bar{x} \equiv \frac{V_{H}}{2}$, then $R\left(\Sigma\left(\theta_{\bar{x}}\right)\right)=r_{B}^{+}(\bar{x})=r_{A}^{-}(\bar{x})$. Since

$$
R\left(\Sigma\left(\theta_{\bar{x}}\right)\right)=r_{B}^{+}(\bar{x})=r_{A}^{-}(\bar{x})>C R^{*}\left(\Sigma\left(\theta_{\bar{x}}\right)\right)
$$

it follows from Theorem 2 of Ellison (2000) that $\Sigma_{S}=\Sigma\left(\theta_{\bar{x}}\right)$; the only stochastically stable outcome is thus $\left\{H H, \frac{V_{H}}{2}, \frac{V_{H}}{2}\right\}$ and the distributional norm is $\left(\frac{V_{H}}{2}, \frac{V_{H}}{2}\right)$.

Acknowledgements This paper was written while Lidia Bagnoli was research fellow of the Department of Economics, University of Bologna; financial support from DSE gratefully acknowledged. We would like to thank Ken Binmore and Kim Hill for comments on an earlier version of this paper. The paper has been presented at a seminar held at the University of Bologna, at the annual meeting of the Italian Economists Society (Matera) and at the ASSET 2012 conference (Lymassol, Cyprus). We thank all participants for their comments; we are the only responsible for errors and imprecisions.

\section{References}

1. Alvard SM (2004) The ultimatum game, fairness and cooperation among big game hunters. In Heinrich et al Foundations of Human Sociality. Economic Experiments and Ethnographic Evidence from Fifteen Small-Scale Societies. Oxford University Press, New York, pp 413-435.

2. Alvard SM, Nolin DA (2002) Rousseau's whale hunt ?. Curr Anthropol 43, 533-559

3. Baker MJ, Swope K (2005) Sharing, gift-giving and optimal resource use incentives in hunter-gatherer societies. Mimeo, Department of Economics, US Naval Academy

4. Bell D (1995) On the nature of sharing: beyond the range of methodological individualism. Curr Anthropol 36, 826-830

5. Binmore K (1998) The evolution of fairness norms. Ration Soc 10, 275-301

6. Binmore K (2007) Economic man or straw man ?. ELSE working paper 262

7. Binmore K, Shaked A (2010) Experimental economics: where next ?. J Econ Behav Organ 73, 87-100

8. Bohem C (2004) What makes humans economically distinctive? A three-species evolutionary comparison and historical analysis. J Bioecon 6, 109-135

9. Boehm C (2012) Moral Origins. The Evolution of Virtue, Altruism, and Shame. Basic Books, New York

10. Chibnik M (2005) Experimental economics in anthropology: a critical assessment. Am Ethnol 32, 198-209

11. Dawid H, MacLeod B (2001) Hold up and the evolution of bargaining conventions. Eur J Econ Soc Syst 15, 139-169

12. Dawid H, MacLeod B (2008) Hold-up and the evolution of investment and bargaining norms. Games Econ Behav 62, 26-52

13. Ellingsen T, Robles J (2002) Does evolution solve the hold-up problem ?. Games Econ Behav 39, 28-53

14. Ellison G (2000) Basin of attraction, long-run stochastic stability, and the speed of step-by-step evolution. Rev Econ Stud 67, 17-45

15. Hackett SC (1993) Incomplete contracting: a laboratory experimental analysis. Econ Inq $31,274-297$ 
16. Hackett SC (1994) Is relational exchange possible in the absence of reputation and repeated contact?. J Law Econ Organ 10, 360-389

17. Hagen EH, Hammerstein P (2006) Game theory and human evolution: a critique of some recent interpretations of experimental games. Theor Popul Biol 69, 339-348

18. Hawkes K (1992) Sharing and collective action. In: Smith E, Winterhalder B (eds) Evolutionary Ecology and Human Behavior, Aldine de Gruyter, New York, pp 269-300.

19. Heinrich et al (2004) Foundations of Human Sociality. Economic Experiments and Ethnographic Evidence from Fifteen Small-Scale Societies. Oxford University Press, New York

20. Hill K (2002) Altruistic cooperation during foraging by the Ache, and the evolved human predisposition to cooperate. Hum Nat 13, 105-128

21. Ichikawa M (1983) An examination of the hunting-dependent life of the Mbuti Pygmies, eastern Zaire. Afr Study Monogr 5, 55-76

22. Kaplan H, Gurven M (2005) The Natural History of Human Food Sharing and Cooperation: A Review and a New Multi-Individual Approach to the Negotiations of Norms, in Gintis et al Moral Sentiments and Material Interests. The Foundations of Cooperation in Economic Life. The MIT Press, Cambridge, pp 75-113.

23. Kaplan H, Hill K (1985) Food sharing among the Ache: tests of explanatory hypotheses. Curr Anthropol 26, 223-246

24. Noldeke G, Samuelson L (1993) An evolutionary analysis of backward and forward induction. Games Econ Behav 5, 425-454

25. Troger T (2002) Why sunk costs matter for bargaining outcomes: an evolutionary approach. J Econ Theory 102, 375-402

26. Woodburn J (1982) Egalitarian Societies. Man 17, 431-451

27. Young P (1993) An evolutionary model of bargaining. J Econ Theory 59, 145-168

28. Young P (1993a) The evolution of conventions. Econometrica 61, 57-84 


\section{Figures captions.}

Fig. 1 Game $\Gamma_{U G}$. Region $H H$ denotes the parameter configuration supporting the unique stochastically stable outcome while region $N N$ denotes the parameter configuration in which no norms evolves since several stochastically stable outcomes can exist

Fig. 2 Game $\Gamma_{D G}$. Region $H H$ denotes the parameter configuration supporting the unique stochastically stable outcome

Fig. 3 Top: game $\Gamma_{D G}$; bottom: game $\Gamma_{U G}$. In both figures the triangle on the right hand side denotes the parameter configuration for which investment by both agents is not efficient. No norms are observed in $\Gamma_{U G}$ while norms coevolve in $\Gamma_{D G}$

Fig. 4 Game $\Gamma_{C}$. Parameter configuration in which efficient investment $(H H)$ and no investment $(L L)$ are stochastically stable, compared with the parameter configuration $(N N)$ where no investment norm evolves 